\title{
The Muskat problem and related topics
}

\author{
Anvarbek Meirmanov and Oleg Galtsev \\ Belgorod State University, Pobedi 85, 308015 Belgorod, Russia \\ (e-mail: anvarbek@list.ru; galtsev_o@bsu.edu.ru)
}

Received March 22, 2018

\begin{abstract}
In the paper, we consider the evolution of the free boundary separating two immiscible viscous fluids with different constant densities in an absolutely rigid solid body and in an elastic skeleton. The motion of the liquids is described by the Stokes equations driven by the input pressure and the force of gravity. For flows in a bounded domain, we prove the existence and uniqueness of classical solutions and emphasize the study of the properties of the moving boundary separating the two fluids.
\end{abstract}

$M S C: 35 Q 35,35 Q 74$

Keywords: Muskat problem, homogenization, Lamé equations, Stokes equations, numerical simulation

\section{Introduction}

It is well known [14] that the Darcy system of filtration, describing the macroscopic flow of an incompressible viscous liquid, is a result of exact homogenization of the Stokes system for an incompressible viscous liquid occupying periodic pore space in an absolutely rigid solid body.

The more complicated macroscopic motion of two immiscible incompressible viscous liquids is governed by the Muskat problem. In this model, one looks for the free boundary $\Gamma(t) \subset Q$ that separates two different domains $Q^{+}(t) \subset Q$ and $Q^{-}(t) \subset Q, Q^{+}(t) \cup \Gamma(t) \cup Q^{-}(t)=Q$, occupied by different fluids. In each of the domains $Q^{ \pm}(t)$ the liquid motion is described by its own Darcy system of filtration, and at the free boundary the normal velocities of the liquids coincide with the normal velocity of the free boundary.

Thus, we may expect that, as in the case of the filtration of a single liquid, the Muskat problem should be a homogenization of the initial boundary value problem for the Stokes system with an inhomogeneous liquid

$$
\mu \Delta \mathbf{u}^{\varepsilon}+g \rho_{\varepsilon} \mathbf{e}=0, \quad \nabla \cdot \mathbf{u}^{\varepsilon}=0, \quad \frac{\mathrm{d} \rho_{\varepsilon}}{\mathrm{d} t}=0,
$$

in a periodic pore space $Q_{\varepsilon}$ of an absolutely rigid solid body $Q$ with the following boundary and initial conditions:

$$
\begin{gathered}
\mathbf{u}^{\varepsilon}(\mathbf{x}, t)=0, \quad \mathbf{x} \in \partial Q_{\varepsilon}, \\
\rho_{\varepsilon}(\mathbf{x}, 0)=\rho_{\varepsilon}^{0}(\mathbf{x}), \quad \mathbf{x} \in Q_{\varepsilon},
\end{gathered}
$$




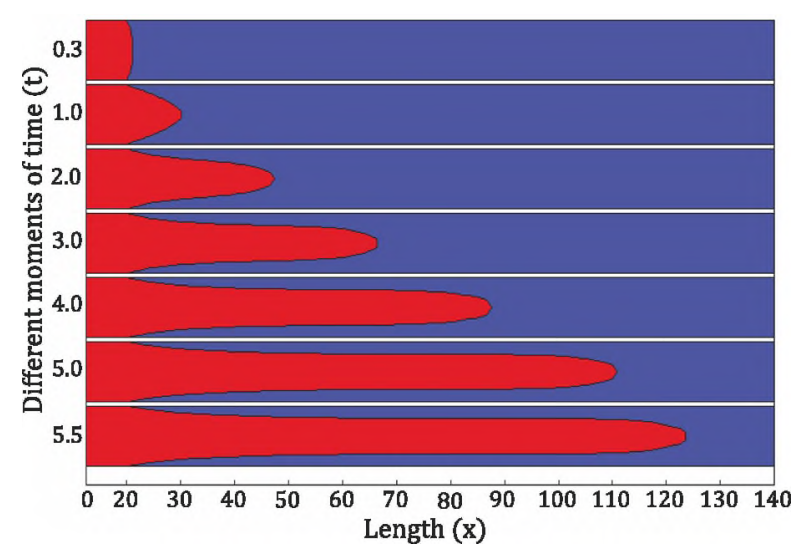

Figure 1. Numerical simulation: successive positions of the free boundary in a single capillary.

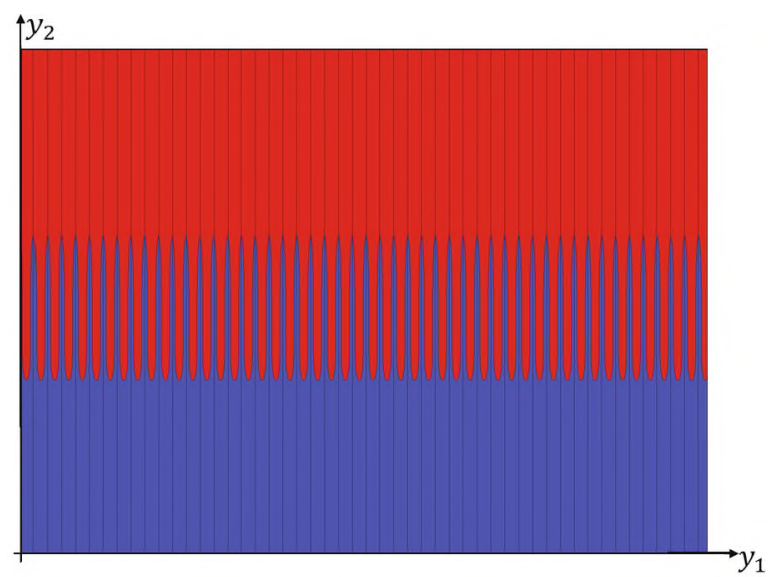

Figure 2. Numerical homogenization for $t=5.5$.

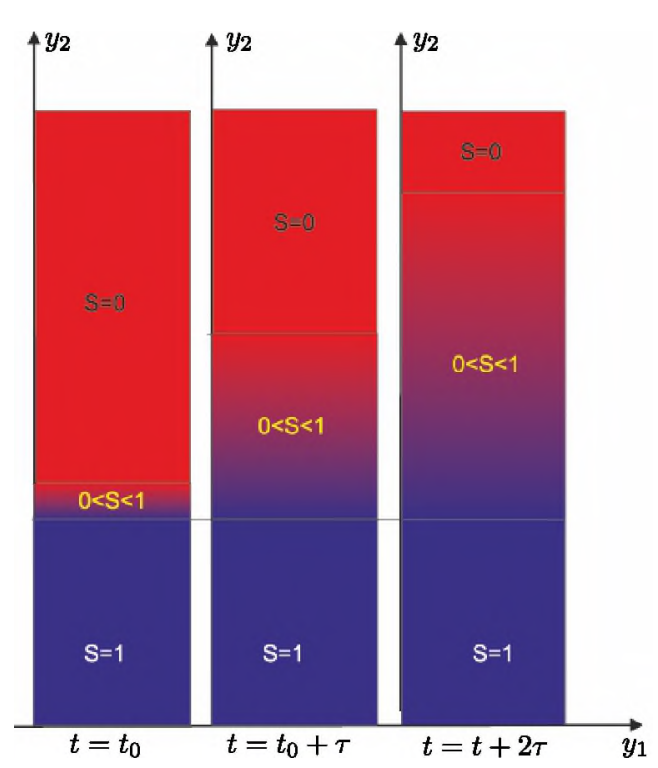

Figure 3. Homogenization by increasing number of capillaries. Concentration of water $s$ for increasing times (left to right).

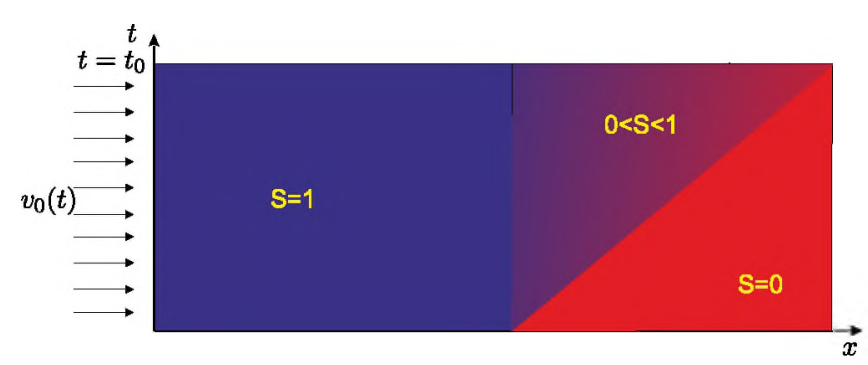

Figure 4. The limit of rigorous numerical upscaling.

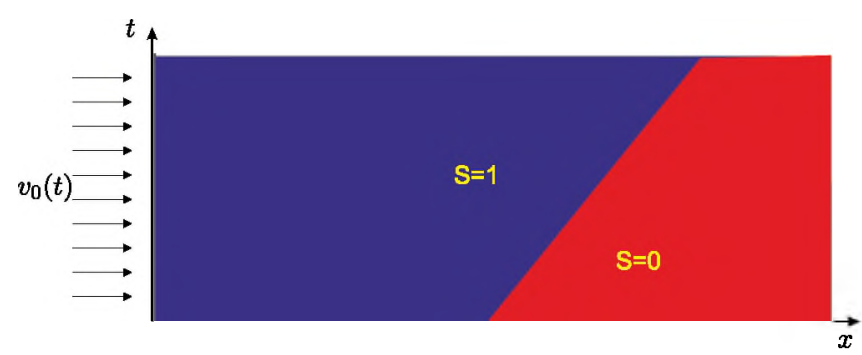

Figure 5. The Muskat problem.

where

$$
\rho_{\varepsilon}^{0}(\mathbf{x})=\rho^{+}=\text {const }, \quad \mathbf{x} \in Q_{\varepsilon}^{+}(0), \quad \rho_{\varepsilon}^{0}(\mathbf{x})=\rho^{-}=\text {const }, \quad \mathbf{x} \in Q_{\varepsilon}^{-}(0),
$$

$\overline{Q_{\varepsilon}^{+}}(0) \cup \overline{Q_{\varepsilon}^{-}}(0)=\overline{Q_{\varepsilon}}, \mu$ is the viscosity, and ge is the acceleration due to gravity.

Due to the boundary condition (1.1), the contact points of the free boundary and the solid skeleton will be permanently fixed at the initial position. Numerical implementations predict the appearance of a water tongue, which grows with time (see Fig. 1). The gradual growth of the number of capillaries (Fig. 2) leads to homogenization of the liquid motion. The domain occupied by the water tongues at a fixed time becomes under homogenization a mushy region, where the concentration $s$ of water varies from 1 to 0 (Figs. 3 and 4).

Now, if we return to the Muskat problem, then we may see that the solution of the Muskat problem corresponding to the macroscopic joint motion of two different liquids has a very simple structure. The free boundary separates two liquids and moves with a constant velocity (Fig. 5). 
So, we cannot obtain the Muskat problem of the liquid motion in the pore space of an absolutely rigid body as a homogenization of the corresponding initial boundary value problem for a Stokes system with an inhomogeneous liquid.

However, if we look for the motion of an inhomogeneous liquid in an elastic solid body, then the situation changes. The contact points of the free boundary and solid body begin to move, and homogenization conserves the free boundary that separates the two liquids [11].

In its simplest setting the problem has the following formulation. Let $Q_{f} \subset Q \subset \mathbb{R}^{2}$, where $Q$ is the unit cube

$$
Q=\left\{\mathbf{x}:-1<x_{i}<1, i=1,2\right\}, \quad \text { and } \quad Q_{f}=\left\{\mathbf{x}:-1<x_{1}<1,-\frac{1}{2}<x_{2}<\frac{1}{2}\right\} .
$$

In dimensionless variables the evolution of the flow is driven by the input pressure and the force of gravity. More precisely, in this problem, we must find the velocity $\mathbf{u}^{f}(\mathbf{x}, t)$, pressure $p_{f}(\mathbf{x}, t)$, and density $\rho_{f}(\mathbf{x}, t)$ of the inhomogeneous liquid in $Q_{f}$, and displacements $\mathbf{u}^{s}(\mathbf{x}, t)$ and pressure $p_{s}(\mathbf{x}, t)$ of an elastic skeleton in $Q_{s}=Q \backslash \overline{Q_{f}}$ from the following system of differential equations:

$$
\begin{aligned}
& \nabla \cdot \mathrm{P}_{f}+\rho_{f} \mathbf{e}=0, \quad \nabla \cdot \mathbf{u}^{f}=0, \quad \mathbf{x} \in Q_{f}, 0<t<T, \\
& \nabla \cdot \mathrm{P}_{s}+\rho_{s} \mathrm{e}=0, \quad \nabla \cdot \mathbf{u}^{s}=0, \quad \mathbf{x} \in Q_{s}, 0<t<T, \\
& \frac{\mathrm{d} \rho_{f}}{\mathrm{~d} t} \equiv \frac{\partial \rho_{f}}{\partial t}+\nabla \cdot\left(\rho_{f} \mathbf{u}^{f}\right)=\frac{\partial \rho_{f}}{\partial t}+\mathbf{u}^{f} \cdot \nabla \rho_{f}=0, \quad \mathbf{x} \in Q_{f}, 0<t<T,
\end{aligned}
$$

where

$$
\begin{gathered}
\mathrm{P}_{f}=2 \mu \mathrm{D}\left(\mathbf{u}^{f}\right)-p_{f} \mathrm{I}, \quad \mathrm{D}\left(\mathbf{u}^{f}\right)=\frac{1}{2}\left(\nabla \mathbf{u}^{f}+\left(\nabla \mathbf{u}^{f}\right)^{*}\right), \\
\mathrm{P}_{s}=2 \lambda \mathrm{D}\left(\mathbf{u}^{s}\right)-p_{s} \mathrm{I}
\end{gathered}
$$

$\mu=$ const is the viscosity of liquids, $\lambda=$ const is the Lamé coefficient, $\mathrm{e}$ is a given vector, $\rho_{s}$ is the density of the solid body, and I is the unit tensor.

The mass and momentum conservation laws dictate the coincidence of velocities and normal tensions of the liquid and solid components:

$$
\mathbf{u}^{f}=\frac{\partial \mathbf{u}^{s}}{\partial t}, \quad \mathrm{P}_{f} \cdot \mathbf{n}=\mathrm{P}_{s} \cdot \mathbf{n}
$$

on the common boundary $S=\partial Q_{f} \cap \partial Q_{s}$ with unit normal vector $\mathbf{n}$. form

The boundary condition on the lateral part $S^{0}=\left\{x_{2}= \pm 1\right\}$ of the boundary $\partial Q$ for $0<t<T$ has the

$$
\mathbf{u}^{s}(\mathbf{x}, t)=0 .
$$

At the "entrance" and "exit" boundaries $S^{ \pm}=\left\{\mathbf{x} \in \partial Q: x_{1}=\mp 1\right\}$,

$$
\begin{aligned}
& \mathrm{P}_{s} \cdot \mathbf{e}_{1}=-p^{+}(\mathbf{x}) \mathbf{e}_{1}, \quad \mathbf{x} \in S_{s}^{+}, \quad \mathrm{P}_{f} \cdot \mathbf{e}_{1}=-p^{+}(\mathbf{x}) \mathbf{e}_{1}, \quad \mathbf{x} \in S_{f}^{+}, \quad 0<t<T, \\
& \mathrm{P}_{s} \cdot \mathbf{e}_{1}=0, \quad \mathbf{x} \in S_{s}^{-}, \quad \mathrm{P}_{f} \cdot \mathbf{e}_{1}=0, \quad \mathbf{x} \in S_{f}^{-}, \quad 0<t<T,
\end{aligned}
$$

where $p^{+}(\mathbf{x})$ is a given function, $S_{f}^{ \pm}=S^{ \pm} \cap \partial Q_{f}, S_{s}^{ \pm}=S^{ \pm} \cap \partial Q_{s}$, and $\mathbf{e}_{i}$ is the unit vector of the $x_{i}$-axis for $i=1,2$.

To simplify our considerations, we pass to the homogeneous boundary conditions at $S^{ \pm}$

$$
\mathrm{P}_{i} \cdot \mathbf{e}_{1}=0, \quad \mathbf{x} \in S_{i}^{ \pm}, i=f, s, 0<t<T,
$$


by introducing the new pressure

$$
p_{f} \rightarrow p_{f}-p^{0}(\mathbf{x}), \quad p^{0}(\mathbf{x})=\frac{1}{2} p^{+}(\mathbf{x})\left(1-x_{1}\right) .
$$

With this new pressure the dynamic equations take the form

$$
\begin{aligned}
& \nabla \cdot \mathrm{P}_{f}+\mathbf{f}+\rho_{f} \mathbf{e}=0, \quad \nabla \cdot \mathbf{u}^{f}=0, \quad \mathbf{x} \in Q_{f}, 0<t<T, \\
& \nabla \cdot \mathrm{P}_{s}+\mathbf{f}=0, \quad \nabla \cdot \mathbf{u}^{s}=0, \quad \mathbf{x} \in Q_{s}, 0<t<T,
\end{aligned}
$$

where

$$
\mathbf{f}(\mathbf{x})=(1-\chi(\mathbf{x})) \rho_{s} \mathbf{e}+\nabla p^{0}(\mathbf{x})
$$

and

$$
\chi(\mathbf{x})=1 \quad \text { for } \mathbf{x} \in Q_{f} \quad \text { and } \quad \chi(\mathbf{x})=0 \quad \text { for } \mathbf{x} \in Q_{s} .
$$

Finally,

$$
\mathbf{u}^{s}(\mathbf{x}, 0)=0, \quad \mathbf{x} \in S^{0} .
$$

The initial and boundary conditions for the density are equivalent to specifying the surface $\Gamma_{0}$ that separates two subdomains $Q_{f}^{ \pm}(0)$ initially occupied by different fluids. For simplicity, we suppose that

$$
\Gamma^{(0)}=\left\{\mathbf{x} \in Q_{f}: x_{1}=h\left(x_{2}\right),-\frac{1}{2}<x_{2}<\frac{1}{2}\right\}
$$

and

$$
-\frac{1}{2}+\delta<h\left(x_{2}\right)<\frac{1}{2}-\delta \text { for }-\frac{1}{2}<x_{2}<\frac{1}{2}
$$

with some $0<\delta<1$.

So, we may expect that the free boundary $\Gamma(t)$ will not touch the given boundaries $S^{ \pm}$, at least for some time interval $0<t<T$.

At the boundaries $S^{ \pm}$for $0<t<T$ and at initial moment $t=0$, the density $\rho_{f}$ is piecewise constant and assumes two positive values characterizing the distinct phases of the flow:

$$
\begin{aligned}
\rho_{f}(\mathbf{x}, t)= & \rho^{ \pm}=\text {const }>0, \quad \mathbf{x} \in S_{f}^{ \pm}, 0<t<T, \\
& \rho_{f}(\mathbf{x}, 0)=\rho_{0}(\mathbf{x}), \quad \mathbf{x} \in Q_{f},
\end{aligned}
$$

where $\rho_{0}(\mathbf{x})=\rho^{ \pm}$for $\mathbf{x} \in Q_{f}^{ \pm}(0)$.

Suppose for simplicity that

$$
\rho^{-} \leqslant \rho_{0}(\mathbf{x}) \leqslant \rho^{+} .
$$

If the velocity $\mathbf{u}^{f}(\mathbf{x}, t)$ is sufficiently smooth, then the Cauchy problem

$$
\frac{\mathrm{d} \mathbf{x}}{\mathrm{d} t}=\mathbf{u}^{f}(\mathbf{x}, t), \quad t>t_{0},\left.\quad \mathbf{x}\right|_{t=t_{0}}=\xi
$$

determines a mapping

$$
\mathbf{x}=\gamma\left(\xi, t ; \mathbf{u}^{f} ; t_{0}\right), \quad \gamma: Q_{f} \rightarrow Q_{f}
$$


In particular, the free boundary $\Gamma(t)$ is determined as the set

$$
\Gamma(t)=\left\{\mathbf{x} \in Q_{f}: \mathbf{x}=\gamma\left(\xi, t ; \mathbf{u}^{f} ; 0\right), \xi \in \Gamma(0)\right\},
$$

and subdomains $Q_{f}^{ \pm}(t)=\left\{\mathbf{x} \in Q_{f}: \rho_{f}(\mathbf{x}, t)=\rho^{ \pm}\right\}$as the sets

$$
\begin{aligned}
Q_{f}^{ \pm}(t)= & \left\{\mathbf{x} \in Q_{f}: \mathbf{x}=\gamma\left(\xi, t ; \mathbf{u}^{f} ; 0\right), \xi \in Q_{f}^{ \pm}(0)\right\} \\
& \cap\left\{\mathbf{x} \in Q_{f}: \mathbf{x}=\gamma\left(\xi, t ; \mathbf{u}^{f} ; t_{0}\right), \xi \in S_{f}^{ \pm}, t_{0}>0\right\} .
\end{aligned}
$$

It is shown that the evolution described by the above equations preserves the existence and uniqueness of two subdomains $Q_{f}^{ \pm}(t)$, each occupied by one of the fluids, that are separated at time $t>0$ by a regular free boundary $\Gamma(t)$. Thus, the problem studied is equivalent to finding $\left\{\mathbf{u}, p_{f}, \mathbf{w}, p_{s}\right\}$ and the moving boundary $\Gamma(t)$.

Theorems on the existence of generalized solutions to the Navier-Stokes system for inhomogeneous incompressible fluids were obtained in, for example, $[2,3,4,5,7,9,10,13,14]$ and [3] (without detailed analysis of a set where the density is discontinuous). The existence and uniqueness of a classical solution to the Stokes equations for an inhomogeneous liquid with Dirichlet boundary conditions have been proved in [1], and with the Neumann boundary conditions in [12]. The weak solutions to problem (1.2)-(1.15) at the microscopic level for arbitrary smooth periodic pore space followed by homogenization has been considered in [10]. Let us call the obtained homogenized free-boundary problem describing the motion of two immiscible incompressible viscous liquids at the macroscopic level as the generalized Muskat problem.

\section{The Muskat problem}

\subsection{A single capillary in an absolutely rigid skeleton: Dirichlet boundary conditions}

We consider a flow of two immiscible viscous fluids with different constant densities in a bounded domain $\Omega \subset \mathbb{R}^{n}$ with $C^{2}$-smooth boundary $S=\partial \Omega$ for dimensions $n \geqslant 2$. The evolution is driven by the force of gravity. The moving boundary, which appears naturally, separates the subdomains occupied by different fluids.

More precisely, the problem is to find the velocity $\mathbf{u}=\left(u_{1}, \ldots, u_{n}\right) \in \mathbb{R}^{n}$, pressure $p \in \mathbb{R}$, and density $\rho \in \mathbb{R}$ from the system of equations for velocity and pressure

$$
\Delta \mathbf{u}=\nabla p+\alpha \rho \mathbf{e}, \quad \nabla \cdot \mathbf{u}=0,
$$

where $\mathrm{e}$ is a given unit vector, and $\alpha=$ const a given scalar constant, and the transport equation for density

$$
\frac{\mathrm{d} \rho}{\mathrm{d} t} \equiv \frac{\partial \rho}{\partial t}+\nabla \cdot(\rho \mathbf{u})=\frac{\partial \rho}{\partial t}+\mathbf{u} \cdot \nabla \rho=0 .
$$

Time $t$ enters the equations for velocity as a parameter, so it needs no initial condition.

The boundary condition on the boundary $S=\partial \Omega$ is

$$
\mathbf{u}=0
$$

At the initial moment $t=0$, the density is piecewise constant and assumes two positive values characterizing the distinct phases of the flow:

$$
\rho(\mathbf{x}, 0)=\rho_{0}(\mathbf{x})=\left\{\begin{array}{ll}
\rho^{+}, & \mathbf{x} \in \Omega^{+}(0), \\
\rho^{-}, & \mathbf{x} \in \Omega^{-}(0),
\end{array} \quad \rho^{ \pm}=\mathrm{const}, \rho^{+}>\rho^{-}>0 .\right.
$$


In this case, the initial condition for the density is equivalent to specifying the surface $\Gamma(0)=\Gamma_{0}$ that separates the two subdomains $\Omega^{ \pm}(0)$ initially occupied by different fluids. We further suppose this surface to be sufficiently regular.

The problem treated here is that of finding $\mathbf{u}(\mathbf{x}, t), p(\mathbf{x}, t)$, and the density $\rho(\mathbf{x}, t)$ from the above equations and the initial and boundary conditions. Note that it is nonlinear because of the coupling term $\mathbf{u} \cdot \nabla \rho$ in (2.2).

We further show that the evolution described by the above equations preserves the existence of two subdomains $\Omega^{ \pm}(t)$, each occupied by one of the fluids, that are separated at time $t>0$ by a regular free boundary $\Gamma(t)$. Thus, the problem studied is equivalent to finding $\mathbf{u}, p$, and the moving boundary $\Gamma(t)$.

Theorems on the existence of generalized solutions to the Navier-Stokes system for inhomogeneous incompressible fluids were obtained in, for example, $[2,3,4,5,13,15,16]$ (without detailed analysis of the set where the density is discontinuous).

Theorem 1. If the initial configuration of the free boundary is a surface $\Gamma_{0}$ of class $C^{2}$, then problem (2.1)(2.4) with piecewise constant initial density has a unique solution in the interval $[0, T]$ of arbitrary length $T>0$. The elements of this solution have the following properties:

(i) For arbitrary $q>n$ and $\lambda=1-n / q$, the velocity satisfies the relation

$$
\mathbf{u} \in L_{\infty}\left(0, T ; W^{2, q}(\Omega)\right) \cap L_{\infty}\left(0, T ; C^{1, \lambda}\right) \cap C^{0, \lambda}\left(0, T ; C^{1, \lambda}\right)
$$

(ii) The free boundary $\Gamma(t)$ is a surface of class $C^{1, \lambda}$ at each time $t \in(0, T]$, and the velocity $V_{\mathbf{n}}(\mathbf{x}, t)$ of the free boundary in the direction of its normal $\mathbf{n}$ at position $\mathbf{x}$ is uniformly bounded,

$$
\sup _{\substack{t \in(0, T) \\ \mathbf{x} \in \Gamma(t)}}\left|V_{\mathbf{n}}(\mathbf{x}, t)\right|<\infty .
$$

(iii) The density has bounded variation,

$$
\rho \in L_{\infty}(0, T ; B V(\Omega)) \cap B V\left(\Omega_{T}\right), \quad \Omega_{T}=\Omega \times(0, T) .
$$

\subsection{A single capillary of an absolutely rigid skeleton: Neumann boundary conditions}

As in the previous subsection, we consider the flow of two immiscible viscous fluids with different constant densities in a single capillary $\Omega=\left\{\mathrm{x} \in \mathbb{R}^{2}:-1<x_{1}<1,-h<x_{2}<h\right\}$. The evolution is driven by input pressure and the force of gravity. More precisely, in this problem, we must find the velocity $\mathbf{u}(\mathbf{x}, t)=$ $\left(u_{1}(\mathbf{x}, t), u_{2}(\mathbf{x}, t)\right) \in \mathbb{R}^{2}$, pressure $p(\mathbf{x}, t)$, and density $\rho(\mathbf{x}, t)$ from the system of equations for velocity and pressure

$$
\begin{gathered}
\mu \Delta \mathbf{u}-\nabla p+g \rho \mathbf{e}=0, \\
\nabla \cdot \mathbf{u}=0,
\end{gathered}
$$

where $\mu=$ const is the viscosity of liquids, e is a given unit vector, and $g$ is the acceleration due to gravity, and the transport equation for the density

$$
\frac{\mathrm{d} \rho}{\mathrm{d} t} \equiv \frac{\partial \rho}{\partial t}+\nabla \cdot(\rho \mathbf{u})=\frac{\partial \rho}{\partial t}+\mathbf{u} \cdot \nabla \rho=0
$$

At the initial moment $t=0$, the density is piecewise constant and assumes two positive values characterizing the distinct phases of the flow:

$$
\rho(\mathbf{x}, 0)=\rho_{0}(\mathbf{x})=\left\{\begin{array}{ll}
\rho^{+}, & \mathbf{x} \in \Omega^{+}(0), \\
\rho^{-}, & \mathbf{x} \in \Omega^{-}(0),
\end{array} \quad \rho^{ \pm}=\mathrm{const}, \rho^{-}>\rho^{+}>0 .\right.
$$

Time $t$ enters the equations for velocity as a parameter, so it needs no initial condition. 
The boundary condition on the lateral part $S^{0}=\left\{\mathbf{x} \in \mathbb{R}^{2}:-1<x_{1}<1, x_{2}= \pm h\right\}$ of the boundary $S=\partial \Omega$ is

$$
\mathbf{u}(\mathbf{x}, t)=0 \text {. }
$$

The boundary condition on the "entrance" $S^{-}=\left\{\mathbf{x} \in \mathbb{R}^{2}: x_{1}=-1,-h<x_{2}<h\right\} \subset S$ and "exit" $S^{+}=$ $\left\{\mathbf{x} \in \mathbb{R}^{2}: x_{1}=1,-h<x_{2}<h\right\} \subset S$ are

$$
\mathrm{P}(\mathbf{u}, p) \cdot \mathbf{n}=-p^{0} \mathbf{n}, \quad \rho=\rho^{ \pm}, \quad \mathbf{x} \in S^{ \pm},
$$

where

$$
\mathrm{P}(\mathbf{u}, p)=2 \mu \mathrm{D}(\mathbf{u})-p \mathrm{I}, \quad \mathrm{D}(\mathbf{u})=\frac{1}{2}\left(\nabla \mathbf{u}+(\nabla \mathbf{u})^{*}\right)
$$

I is the unit tensor, $p^{0}(\mathbf{x})$ is a given function, and $\mathbf{n}=(1,0)$ is the unit normal vector to $S^{ \pm}$. Note that we need the boundary condition for the density only at points $\mathbf{x} \in S^{ \pm}$where $\pm \mathbf{u} \cdot \mathbf{n}<0$.

The initial and boundary conditions for the density are equivalent to specifying the surface $\Gamma_{0}$ that separates the two subdomains $\Omega^{ \pm}(0)$ initially occupied by different fluids. For simplicity, we suppose that $\Gamma^{(0)}=$ $\left\{\mathbf{x} \in \Omega: x_{1}=0,-h<x_{2}<h\right\}, \Omega^{ \pm}=\left\{\mathbf{x} \in \Omega: 0< \pm x_{1}<1\right\}$.

If the velocity $\mathbf{u}(\mathbf{x}, t)$ is sufficiently smooth, then the Cauchy problem

$$
\frac{\mathrm{d} \mathbf{x}}{\mathrm{d} t}=\mathbf{u}(\mathbf{x}, t), \quad t>t_{0},\left.\quad \mathbf{x}\right|_{t=t_{0}}=\xi
$$

determines a mapping

$$
\mathbf{x}=\gamma\left(\xi, t ; \mathbf{u} ; t_{0}\right), \quad \gamma: \bar{\Omega} \rightarrow \bar{\Omega} .
$$

In particular, the free boundary $\Gamma(t)$ is determined as the set

$$
\Gamma(t)=\left\{\mathbf{x} \in \Omega: \mathbf{x}=\gamma(\xi, t ; \mathbf{u} ; 0), \xi \in \Gamma^{(0)}\right\}
$$

and subdomains $\Omega^{ \pm}(t)=\left\{\mathbf{x} \in \Omega: \rho(\mathbf{x}, t)=\rho^{ \pm}\right\}$as the sets

$$
\begin{aligned}
\Omega^{ \pm}(t)= & \left\{\mathbf{x} \in \Omega: \mathbf{x}=\gamma(\xi, t ; \mathbf{u} ; 0), \xi \in \Omega^{ \pm}\right\} \\
& \cap\left\{\mathbf{x} \in \Omega: \mathbf{x}=\gamma\left(\xi, t ; \mathbf{u} ; t_{0}\right), \xi \in S^{ \pm}(0), t_{0}>0\right\} .
\end{aligned}
$$

The problem treated here is that of finding the velocity $\mathbf{u}(\mathbf{x}, t)$, pressure $p(\mathbf{x}, t)$, and density $\rho(\mathbf{x}, t)$ from the above equations and initial and boundary data. Note that it is nonlinear because of the coupling term $\mathbf{u} \cdot \nabla \rho$ in (2.6).

To simplify our considerations, we pass to the homogeneous boundary conditions

$$
\mathrm{P}(\mathbf{u}, p) \cdot \mathbf{n}=0, \quad \mathbf{x} \in S^{ \pm}
$$

by introducing a new pressure $p \rightarrow p-p^{0}(\mathbf{x})$ :

$$
\mu \Delta \mathbf{u}-\nabla p=\mathbf{f} \equiv \nabla p^{0}-g \rho \mathbf{e}
$$

where $\nabla p^{0}$ is a bounded function:

$$
\left|\nabla p^{0}(\mathbf{x}, t)\right|<P^{0}=\text { const. }
$$

We further show that the evolution described by the above equations preserves the existence of two subdomains $\Omega^{ \pm}(t)$, each occupied by one of the fluids, that are separated at time $t>0$ by a regular free boundary $\Gamma(t)$. Thus, the problem studied is equivalent to finding $\mathbf{u}, p$, and the moving boundary $\Gamma(t)$.

Let $\Omega^{(m)}=\left\{\mathbf{x} \in \Omega:-1+1 / m<x_{1}<1-1 / m\right\}$, where $m>1$ (i.e., any real positive number). 
Theorem 2. Under condition (2.12), problem (2.5)-(2.8), (2.10)-(2.11) has a unique solution in the interval $[0, T)$ for some $T>0$. The elements of this solution have the following properties:

(i) For arbitrary positive $m \in \mathbb{N}, q>2$, and $\lambda=1-2 / q$, the velocity $\mathbf{u}$ and pressure $p$ satisfy the regularity conditions

$$
\mathbf{u} \in L_{\infty}\left(0, T ; W^{2, q}\left(\Omega^{(m)}\right)\right) \cap L_{\infty}\left(0, T ; C^{1, \lambda}\left(\Omega^{(m)}\right)\right), \quad p \in L_{\infty}\left(0, T ; W^{1, q}\left(\Omega^{(m)}\right)\right),
$$

equations (2.5), (2.11) almost everywhere in $\Omega_{T}=\Omega \times(0, T)$, boundary condition (2.8) in the usual sense, and boundary condition (2.9) in the sense of distributions as the integral identity

$$
\int_{\Omega}(\mathrm{P}(\mathbf{u}(t), p(t)): \mathrm{D}(\varphi)+\mathbf{f} \cdot \varphi) \mathrm{d} x=0
$$

for almost all $0<t<T$ and for any smooth solenoidal functions $\varphi$ vanishing at $\mathbf{x} \in S^{0}$.

(ii) The free boundary $\Gamma(t)$ is a surface of class $C^{1, \lambda}$ at each time $t \in[0, T)$, and the normal velocity $V_{n}(x, t)$ of the free boundary in the direction of its normal $\mathbf{n}$ at position $\mathrm{x}$ is uniformly bounded,

$$
\sup _{\substack{t \in(0, T) \\ \mathbf{x} \in \Gamma(t)}}\left|V_{n}(\mathbf{x}, t)\right|<\infty
$$

(iii) The density $\rho$ has bounded variation,

$$
\rho \in L_{\infty}\left(0, T ; B V\left(\Omega^{(m)}\right)\right) \cap B V\left(\Omega_{T}^{(m)}\right),
$$

and satisfies the transport equation (2.5) in the sense of distributions

$$
\int_{\Omega_{T}} \rho\left(\frac{\partial \psi}{\partial t}+\mathbf{u} \cdot \nabla \psi\right) \mathrm{d} x \mathrm{~d} t=-\int_{\Omega} \rho_{0}(\mathbf{x}) \psi(\mathbf{x}, 0) \mathrm{d} x
$$

for any smooth functions $\psi$ vanishing at $t=T$ and $\mathrm{x} \in S^{ \pm}$.

The time $T$ of the existence of the classical solution depends on the behavior of the free boundary $\Gamma(t)$. Namely, let $\delta^{ \pm}(t)$ be the distance between $\Gamma(t)$ and the boundary $S^{ \pm}$, and let $\delta(t)=\min \left(\delta^{-}(t), \delta^{+}(t)\right)$. Then $\delta(t)>0$ for all $0<t<T$ and $\delta(t) \rightarrow 0$ as $t \rightarrow T$.

We use the standard notations of functional spaces and norm there.

\subsection{A single capillary of an elastic skeleton: Neumann boundary conditions}

We consider a flow of two immiscible viscous fluids with different constant densities in a single capillary $Q_{f} \subset Q \subset \mathbb{R}^{2}$, where $Q$ is a unit cube. Suppose for simplicity that

$$
Q=\left\{\mathbf{x}:-1<x_{i}<1, i=1,2\right\}, \quad Q_{f}=\left\{\mathrm{x}:-1<x_{1}<1,-\frac{1}{2}<x_{2}<\frac{1}{2}\right\} .
$$

In dimensionless variables the evolution of flow is driven by the input pressure and the force of gravity. More precisely, in this problem, we have to find the velocity $\mathbf{u}^{f}(\mathbf{x}, t)$, pressure $p_{f}(\mathbf{x}, t)$, and density $\rho_{f}(\mathbf{x}, t)$ of the 
inhomogeneous liquid in $Q_{f}$, and the displacements $\mathbf{u}^{s}(\mathbf{x}, t)$ and pressure $p_{s}(\mathbf{x}, t)$ of an elastic skeleton in $Q_{s}=Q \backslash \overline{Q_{f}}$ from the following system of differential equations:

$$
\begin{aligned}
& \nabla \cdot \mathrm{P}_{f}+\rho_{f} \mathbf{e}=0, \quad \nabla \cdot \mathbf{u}^{f}=0, \quad \mathbf{x} \in Q_{f}, 0<t<T, \\
& \nabla \cdot \mathrm{P}_{s}+\rho_{s} \mathrm{e}=0, \quad \nabla \cdot \mathbf{u}^{s}=0, \quad \mathbf{x} \in Q_{s}, 0<t<T, \\
& \frac{\mathrm{d} \rho_{f}}{\mathrm{~d} t} \equiv \frac{\partial \rho_{f}}{\partial t}+\nabla \cdot\left(\rho_{f} \mathbf{u}^{f}\right)=\frac{\partial \rho_{f}}{\partial t}+\mathbf{u}^{f} \cdot \nabla \rho_{f}=0, \quad \mathbf{x} \in Q_{f}, 0<t<T,
\end{aligned}
$$

where

$$
\begin{gathered}
\mathrm{P}_{f}=2 \mu \mathrm{D}\left(\mathbf{u}^{f}\right)-p_{f} \mathrm{I}, \quad \mathrm{D}\left(\mathbf{u}^{f}\right)=\frac{1}{2}\left(\nabla \mathbf{u}^{f}+\left(\nabla \mathbf{u}^{f}\right)^{*}\right), \\
\mathrm{P}_{s}=2 \lambda \mathrm{D}\left(\mathbf{u}^{s}\right)-p_{s} \mathrm{I},
\end{gathered}
$$

$\mu=$ const is the viscosity of liquids, $\lambda=$ const is the Lamé coefficient, e is a given vector, $\rho_{s}$ is the density of the solid body, and $\mathrm{I}$ is the unit tensor.

The mass and momentum conservation laws dictate the coincidence of velocities and normal tensions in the liquid and solid components

$$
\mathbf{u}^{f}=\frac{\partial \mathbf{u}^{s}}{\partial t}, \quad \mathrm{P}_{f} \cdot \mathbf{n}=\mathrm{P}_{s} \cdot \mathbf{n}
$$

on the common boundary $S=\partial Q_{f} \cap \partial Q_{s}$ with unit normal vector $\mathbf{n}$.

The boundary condition on the lateral part $S^{0}=\left\{x_{2}= \pm 1\right\}$ of the boundary $\partial Q$ for $0<t<T$ has the form

$$
\mathbf{u}^{s}(\mathbf{x}, t)=0 .
$$

At the "entrance" and "exit" boundaries $S^{ \pm}=\left\{\mathbf{x} \in \partial Q: x_{1}=\mp 1\right\}$,

$$
\begin{aligned}
& \mathrm{P}_{s} \cdot \mathbf{e}_{1}=-p^{+}(\mathbf{x}) \mathbf{e}_{1}, \quad \mathbf{x} \in S_{s}^{+}, \quad \mathrm{P}_{f} \cdot \mathbf{e}_{1}=-p^{+}(\mathbf{x}) \mathbf{e}_{1}, \quad \mathbf{x} \in S_{f}^{+}, \quad 0<t<T, \\
& \mathrm{P}_{s} \cdot \mathbf{e}_{1}=0, \quad \mathbf{x} \in S_{s}^{-}, \quad \mathrm{P}_{f} \cdot \mathbf{e}_{1}=0, \quad \mathbf{x} \in S_{f}^{-}, \quad 0<t<T,
\end{aligned}
$$

where $p^{+}(\mathbf{x})$ is a given function, $S_{f}^{ \pm}=S^{ \pm} \cap \partial Q_{f}, S_{s}^{ \pm}=S^{ \pm} \cap \partial Q_{s}$, and $\mathbf{e}_{i}$ is the unit vector of the $x_{i}$-axis for $i=1,2$.

To simplify our considerations, we pass to the homogeneous boundary conditions at $S^{ \pm}$

$$
\mathrm{P}_{i} \cdot \mathbf{e}_{1}=0, \quad \mathbf{x} \in S_{i}^{ \pm}, i=f, s, 0<t<T,
$$

by introducing the new pressure

$$
p_{f} \rightarrow p_{f}-p^{0}(\mathbf{x}), \quad p^{0}(\mathbf{x})=\frac{1}{2} p^{+}(\mathbf{x})\left(1-x_{1}\right) .
$$

With this new pressure the dynamics equations take the form

$$
\begin{aligned}
& \nabla \cdot \mathbf{P}_{f}+\mathbf{f}+\rho_{f} \mathbf{e}=0, \quad \nabla \cdot \mathbf{u}^{f}=0, \quad \mathbf{x} \in Q_{f}, 0<t<T \\
& \nabla \cdot \mathbf{P}_{s}+\mathbf{f}=0, \quad \nabla \cdot \mathbf{u}^{s}=0, \quad \mathbf{x} \in Q_{s}, 0<t<T
\end{aligned}
$$

where

$$
\mathbf{f}(\mathbf{x})=(1-\chi(\mathbf{x})) \rho_{s} \mathbf{e}+\nabla p^{0}(\mathbf{x})
$$


and

$$
\chi(\mathbf{x})=1 \quad \text { for } \mathbf{x} \in Q_{f} \quad \text { and } \quad \chi(\mathbf{x})=0 \quad \text { for } \mathbf{x} \in Q_{s}
$$

Finally,

$$
\mathbf{u}^{\mathrm{s}}(\mathbf{x}, 0)=0, \quad \mathbf{x} \in S^{0} .
$$

The initial and boundary conditions for density are equivalent to specifying the surface $\Gamma_{0}$ that separates two subdomains $Q_{f}^{ \pm}(0)$ initially occupied by different fluids. For simplicity, we suppose that

$$
\Gamma^{(0)}=\left\{\mathbf{x} \in Q_{f}: x_{1}=h\left(x_{2}\right),-\frac{1}{2}<x_{2}<\frac{1}{2}\right\}
$$

and

$$
-\frac{1}{2}+\delta<h\left(x_{2}\right)<\frac{1}{2}-\delta \quad \text { for }-\frac{1}{2}<x_{2}<\frac{1}{2}
$$

with some $0<\delta<1$.

So, we may expect that the free boundary $\Gamma(t)$ will not touch the given boundaries $S^{ \pm}$at least for some time interval $0<t<T$.

At the boundaries $S^{ \pm}$for $0<t<T$ and at initial moment $t=0$, the density $\rho_{f}$ is piecewise constant and assumes two positive values characterizing the distinct phases of the flow

$$
\begin{aligned}
\rho_{f}(\mathbf{x}, t)= & \rho^{ \pm}=\mathrm{const}>0, \quad \mathbf{x} \in S_{f}^{ \pm}, 0<t<T, \\
& \rho_{f}(\mathbf{x}, 0)=\rho_{0}(\mathbf{x}), \quad \mathbf{x} \in Q_{f},
\end{aligned}
$$

where $\rho_{0}(\mathbf{x})=\rho^{ \pm}$for $\mathbf{x} \in Q_{f}^{ \pm}(0)$.

Suppose for simplicity that

$$
\rho^{-} \leqslant \rho_{0}(\mathbf{x}) \leqslant \rho^{+} .
$$

If the velocity $\mathbf{u}^{f}(\mathbf{x}, t)$ is sufficiently smooth, then the Cauchy problem

$$
\frac{\mathrm{d} \mathbf{x}}{\mathrm{d} t}=\mathbf{u}^{f}(\mathbf{x}, t), \quad t>t_{0},\left.\quad \mathbf{x}\right|_{t=t_{0}}=\xi
$$

determines a mapping

$$
\mathbf{x}=\gamma\left(\xi, t ; \mathbf{u}^{f} ; t_{0}\right), \quad \gamma: Q_{f} \rightarrow Q_{f}
$$

In particular, the free boundary $\Gamma(t)$ is determined as the set

$$
\Gamma(t)=\left\{\mathbf{x} \in Q_{f}: \mathbf{x}=\gamma\left(\xi, t ; \mathbf{u}^{f} ; 0\right), \xi \in \Gamma(0)\right\},
$$

and subdomains $Q_{f}^{ \pm}(t)=\left\{\mathbf{x} \in Q_{f}: \rho_{f}(\mathbf{x}, t)=\rho^{ \pm}\right\}$as the sets

$$
\begin{aligned}
Q_{f}^{ \pm}(t)= & \left\{\mathbf{x} \in Q_{f}: \mathbf{x}=\gamma\left(\xi, t ; \mathbf{u}^{f} ; 0\right), \xi \in Q_{f}^{ \pm}(0)\right\} \\
& \cap\left\{\mathbf{x} \in Q_{f}: \mathbf{x}=\gamma\left(\xi, t ; \mathbf{u}^{f} ; t_{0}\right), \xi \in S_{f}^{ \pm}, t_{0}>0\right\} .
\end{aligned}
$$

The problem treated here is that of finding the velocity $\mathbf{u}^{f}(\mathbf{x}, t)$ and pressure $p_{f}(\mathbf{x}, t)$ of the liquid in pores, the displacement $\mathbf{u}^{s}(\mathbf{x}, t)$ and pressure $p_{s}(\mathbf{x}, t)$ of the solid skeleton, and the density $\rho_{f}(\mathbf{x}, t)$ of the liquid 
from the above equations and initial and boundary data. Note that it is nonlinear because of the coupling term $\mathbf{u}^{f} \cdot \nabla \rho_{f}$ in (2.13).

We further show that the evolution described by the above equations preserves the existence of two subdomains $Q_{f}^{ \pm}(t)$, each occupied by one of the fluids, that are separated at time $t>0$ by a regular free boundary $\Gamma(t)$. Thus, the problem studied is equivalent to finding $\left\{\mathbf{u}^{f}, p_{f}, \mathbf{u}^{s}, p_{s}\right\}$ and the moving boundary $\Gamma(t)$.

We use the standard notations of functional spaces and norm there.

Theorem 3. Under the conditions

$$
\|\mathbf{f}\|_{\infty, Q}=C_{0}<\infty, \quad \Gamma(0) \in C^{1+\alpha}, \quad 0<\alpha<1
$$

problem (2.13)-(2.19) has a unique solution in the interval $[0, T)$ for some $T>0$.

The elements of this solution possess the following properties:

(i) For any $0<\delta<1$ and $0<\alpha<1$, the velocity $\mathbf{u}$ and pressure $p$ satisfy the regularity conditions

$$
\mathbf{u} \in L_{\infty}\left(0, T ; W_{2}^{3}\left(Q^{(\delta)}\right)\right) \cap L_{\infty}\left(0, T ; C^{1+\alpha}\left(Q^{(\delta)}\right)\right), \quad p \in L_{\infty}\left(0, T ; W_{2}^{2}\left(Q^{(\delta)}\right)\right),
$$

equations (2.17) almost everywhere in $Q \times(0, T)$, boundary conditions (2.15), (2.18), and initial conditions (2.17) and (2.19) in the usual sense, and boundary conditions (2.14) and (2.16) in the sense of distributions as the integral identity

$$
\int_{\Omega}(\mathrm{P}(\mathbf{u}(t), p(t)): \mathrm{D}(\varphi)+\mathbf{f} \cdot \varphi) \mathrm{d} x=0
$$

for almost all $0<t<T$ and for any smooth solenoidal functions $\varphi$ vanishing at $\mathbf{x} \in S^{0}$.

(ii) The free boundary $\Gamma(t)$ is a surface of class $C^{1, \alpha}$ at each time $t \in[0, T)$, and the normal velocity $V_{n}(\mathbf{x}, t)$ of the free boundary in the direction of its normal $\mathbf{n}$ at position $\mathbf{x}$ is uniformly bounded,

$$
\sup _{\substack{t \in(0, T) \\ \mathbf{x} \in \Gamma(t)}}\left|V_{n}(\mathbf{x}, t)\right|<\infty .
$$

(iii) The density $\rho$ has bounded variation,

$$
\rho \in L_{\infty}\left(0, T ; B V\left(Q^{(\delta)}\right)\right) \cap B V\left(Q^{(\delta)} \times(0, T)\right)
$$

and satisfies the transport equation (2.13) in the sense of distributions

$$
\int_{\Omega_{T}} \rho\left(\frac{\partial \psi}{\partial t}+u \cdot \nabla \psi\right) \mathrm{d} x \mathrm{~d} t=-\int_{\Omega} \rho_{0}(\mathbf{x}) \psi(\mathbf{x}, 0) \mathrm{d} x
$$

for any smooth functions $\psi$, vanishing at $t=T$ and $\mathrm{x} \in S^{ \pm}$.

The time $T$ of the existence of the classical solution depends on the behavior of the free boundary $\Gamma(t)$. Namely, let $\delta^{ \pm}(t)$ be the distance between $\Gamma(t)$ and the boundary $S^{ \pm}$, and let $\delta(t)=\min \left(\delta^{-}(t), \delta^{+}(t)\right)$. Then $\delta(t)>0$ for all $0<t<T$ and $\delta(t) \rightarrow 0$ as $t \rightarrow T$.

Because of the restriction of the volume of the paper, we prove only Theorem 3. 


\subsubsection{Proof of Theorem 3}

We divide the proof of Theorem 3 into several steps.

1. First, we show that the problem of finding $u$ and $\rho$ has at least one classical solution for an approximate smooth initial density $\rho_{0}^{\varepsilon} \in C^{\infty}\left(Q_{f}\right)$.

2. Next, we specify a class of functions with certain regularity properties and, using a compactness argument, establish the convergence of smooth solutions to the solution of the original problem with piecewise constant initial density $\rho_{0}$.

3. Finally, we prove the existence of a smooth surface separating the parts of the domain occupied by two different fluids.

In what follows, by $C$ we denote constants depending only on $C_{0}, \mathbf{f}, \rho^{ \pm}$, and $\rho_{s}$.

\subsubsection{Approximate smooth density}

Throughout this subsection, we fix the initial density $\rho_{0}^{\varepsilon} \in C^{\infty}\left(Q_{f}\right), \varepsilon>0$.

More precisely, we put

$$
\rho_{0}^{\varepsilon}(\mathbf{x})=\mathbf{M}_{\varepsilon}^{(2)}\left(\rho_{0}\right)=\frac{1}{\varepsilon^{2}} \int_{Q_{f}} J\left(\frac{|\mathbf{x}-\mathbf{y}|}{\varepsilon}\right) \rho_{0}(\mathbf{y}) \mathrm{d} y
$$

for $\mathbf{x} \in Q_{f}$, where

$$
\begin{gathered}
J(s) \geqslant 0, \quad J(s)=0 \quad \text { for }|s|>1, \quad J(s)=J(-s), \\
J \in C^{\infty}(-\infty,+\infty), \quad \int_{\mathbb{R}^{2}} J(|x|) \mathrm{d} x=1,
\end{gathered}
$$

and consider the following approximate problem:

$$
\begin{aligned}
& \nabla \cdot \mathrm{P}_{f}^{\varepsilon}+\rho_{f}^{\varepsilon} \mathbf{e}+\mathbf{f}=0, \quad \nabla \cdot \mathbf{u}^{f, \varepsilon}=0, \quad \mathbf{x} \in Q_{f}, 0<t<T, \\
& \nabla \cdot \mathrm{P}_{s}^{\varepsilon}+\mathbf{f}=0, \quad \nabla \cdot \mathbf{u}^{s, \varepsilon}=0, \quad \mathbf{x} \in Q_{s}, 0<t<T, \quad \mathbf{u}^{s, \varepsilon}(\mathbf{x}, 0)=0, \quad \mathbf{x} \in S, \\
& \mathbf{u}^{f, \varepsilon}=\frac{\partial \mathbf{u}^{s, \varepsilon}}{\partial t}, \quad \mathrm{P}_{f}^{\varepsilon} \cdot \mathbf{n}=\mathrm{P}_{s}^{\varepsilon} \cdot \mathbf{n}, \quad \mathbf{x} \in S, 0<t<T, \\
& \mathrm{P}_{i}^{\varepsilon} \cdot \mathbf{e}_{1}=0, \quad \mathbf{x} \in S_{i}^{ \pm}, i=f, s, \quad \mathbf{u}^{s, \varepsilon}(\mathbf{x}, t)=0, \quad \mathbf{x} \in S^{0}, 0<t<T, \\
& \mathrm{P}_{f}\left(\mathbf{u}^{f, \varepsilon}, p_{f}^{\varepsilon}\right)=2 \mu \mathrm{D}\left(\mathbf{u}^{f, \varepsilon}\right)-p_{f}^{\varepsilon} \mathrm{I}, \quad \mathrm{P}_{s}\left(\mathbf{u}^{s, \varepsilon}, p_{s}^{\varepsilon}\right)=2 \lambda \mathrm{D}\left(\mathbf{u}^{s, \varepsilon}\right)-p_{s}^{\varepsilon} \mathrm{I}, \\
& \frac{\partial \rho^{\varepsilon}}{\partial t}+\mathbf{v}^{\varepsilon} \cdot \nabla \rho^{\varepsilon}=0, \quad \mathbf{x} \in Q, 0<t<T, \quad \rho^{\varepsilon}(\mathbf{x}, t)=\rho_{0}^{ \pm}, \quad \mathbf{x} \in S_{f}^{ \pm}, 0<t<T, \\
& \rho^{\varepsilon}(\mathbf{x}, 0)=\rho_{0}^{\varepsilon}(\mathbf{x}), \quad \mathbf{x} \in Q, \quad \mathbf{v}^{\varepsilon}=\mathbf{M}_{\varepsilon}^{(1)}\left(\mathbf{M}_{\varepsilon}^{(2)}\left(\mathbf{u}^{f, \varepsilon}\right)\right) .
\end{aligned}
$$

Here

$$
\mathbf{M}_{\varepsilon}^{(1)}(\mathbf{v})=\frac{1}{\varepsilon} \int_{0}^{\infty} J\left(\frac{|t-\tau|}{\varepsilon}\right) \mathbf{v}(\mathbf{x}, \tau) \mathrm{d} \tau
$$


DEFINITION 1. We say that a set of functions $\left\{\mathbf{u}^{f, \varepsilon}, p_{f}^{\varepsilon}, \mathbf{u}^{s, \varepsilon}, p_{s}^{\varepsilon}, \rho^{\varepsilon}\right\}$

$$
\begin{gathered}
\mathbf{u}^{i, \varepsilon} \in L_{\infty}\left((0, T) ; W_{2}^{1}\left(Q_{i}\right)\right), \quad p_{i}^{\varepsilon} \in L_{\infty}\left((0, T) ; L_{2}\left(Q_{i}\right)\right), \quad i=f, s, \\
\rho^{\varepsilon} \in C^{1}\left(\overline{Q_{T}}\right), \quad Q_{T}=Q \times(0, T),
\end{gathered}
$$

is a weak solution of problem (2.20), (2.21) if it satisfies the integral identity

$$
\begin{aligned}
\int_{Q_{f}} & \mathrm{P}_{f}\left(\mathbf{u}^{f, \varepsilon}, p_{f}^{\varepsilon}\right): \mathrm{D}(\varphi) \mathrm{d} x+\int_{Q_{s}} \mathrm{P}_{s}\left(\mathbf{u}^{s, \varepsilon}, p_{s}^{\varepsilon}\right): \mathrm{D}(\varphi) \mathrm{d} x \\
= & \int_{Q_{f}} \rho^{\varepsilon}(\mathbf{e} \cdot \varphi) \mathrm{d} x+\int_{Q} \mathbf{f} \cdot \varphi \mathrm{d} x
\end{aligned}
$$

for almost all $t \in(0, T)$ and for arbitrary smooth functions $\varphi(\mathbf{x})$ vanishing at $S^{0}$, and problem (2.21) in the usual sense.

To solve problem (2.20)-(2.21), we use the Schauder fixed point theorem [6].

Let $\mathcal{M}$ be the set of all continuous functions

$$
\tilde{\rho} \in C(\bar{G}), \quad G=Q_{f} \times(0, T),
$$

such that

$$
\rho^{-} \leqslant \widetilde{\rho}(\mathbf{x}, t) \leqslant \rho^{+} .
$$

For fixed $\varepsilon>0$, we define the nonlinear operator

$$
\Phi: \mathcal{M} \rightarrow \mathcal{M}, \quad \rho=\Phi(\widetilde{\rho})
$$

where $\left\{\mathbf{u}^{f}, p_{f}, \mathbf{u}^{s}, p_{s}, \rho\right\}$ is a weak solution of the nonlinear boundary-value problem

$$
\begin{aligned}
& \nabla \cdot \mathbf{P}_{f}+\tilde{\rho}^{\varepsilon} \mathbf{e}+\mathbf{f}=0, \quad \nabla \cdot \mathbf{u}^{f}=0, \quad \mathbf{x} \in Q_{f}, 0<t<T, \\
& \nabla \cdot \mathbf{P}_{s}+\mathbf{f}=0, \quad \nabla \cdot \mathbf{u}^{s}=0, \quad \mathbf{x} \in Q_{s}, 0<t<T, \quad \mathbf{u}^{s}(\mathbf{x}, 0)=0, \quad \mathbf{x} \in S, \\
& \mathbf{u}^{f}=\frac{\partial \mathbf{u}^{s}}{\partial t}, \quad \mathbf{P}_{f} \cdot \mathbf{n}=\mathbf{P}_{s} \cdot \mathbf{n}, \quad \mathbf{x} \in S, 0<t<T, \\
& \mathrm{P}_{i} \cdot \mathbf{e}_{1}=0, \quad \mathbf{x} \in S_{i}^{ \pm}, i=f, s, \quad \mathbf{u}^{s}(\mathbf{x}, t)=0, \quad \mathbf{x} \in S^{0}, 0<t<T, \\
& \frac{\partial \rho}{\partial t}+\mathbf{v}^{\varepsilon} \cdot \nabla \rho=0, \quad \mathbf{x} \in Q, 0<t<T, \\
& \rho(\mathbf{x}, t)=\rho_{0}^{ \pm}, \quad \mathbf{x} \in S_{f}^{ \pm}, 0<t<T, \quad \rho(\mathbf{x}, 0)=\rho_{0}^{\varepsilon}(\mathbf{x}), \quad \mathbf{x} \in Q,
\end{aligned}
$$

where

$$
\begin{aligned}
& \widehat{\rho}^{\varepsilon}(\mathbf{x}, t)=\mathbf{M}_{\varepsilon}^{(1)}(\tilde{\rho}), \quad \mathbf{v}^{\varepsilon}=\mathbf{M}_{\varepsilon}^{(1)}\left(\mathbf{M}_{\varepsilon}^{(2)}\left(u^{f}\right)\right), \\
& \mathrm{P}_{f}=2 \mu \mathrm{D}\left(\mathbf{u}^{f}\right)-p_{f} \mathrm{I}, \quad \mathrm{P}_{s}=2 \lambda \mathrm{D}\left(\mathbf{u}^{s}\right)-p_{s} \mathrm{I} .
\end{aligned}
$$

The properties of the mollifier $\mathbf{M}_{\varepsilon}^{(2)}$ and continuity equation for $u$ imply the continuity equation for $\mathbf{v}^{\varepsilon}$ :

$$
\nabla \cdot \mathbf{v}^{\varepsilon}=0, \quad \mathbf{x} \in Q_{f}, 0<t<T .
$$


Lemma 1. Under the conditions of Theorem 3, problem (2.24) has a unique weak solution

$$
\mathbf{u}^{i} \in L_{\infty}\left((0, T) ; W_{2}^{1}\left(Q_{i}\right)\right), \quad i=f, s, \quad \frac{\partial \mathbf{u}^{s}}{\partial t} \in L_{2}\left((0, T) ; W_{2}^{1}\left(Q_{s}\right)\right),
$$

satisfying the following estimates:

$$
\begin{gathered}
\int_{0}^{T} \int_{Q_{f}} \mathrm{D}\left(\mathbf{u}^{f}\right): \mathrm{D}\left(\mathbf{u}^{f}\right) \mathrm{d} x \mathrm{~d} t+\max _{0<t<T} \int_{Q_{s}} \mathrm{D}\left(\mathbf{u}^{s}(\mathbf{x}, t)\right): \mathrm{D}\left(\mathbf{u}^{s}(\mathbf{x}, t)\right) \mathrm{d} x \leqslant C, \\
\max _{0<t<T} \int_{Q_{f}} \mathrm{D}\left(\mathbf{u}^{f}(\mathbf{x}, t)\right): \mathrm{D}\left(\mathbf{u}^{f}(\mathbf{x}, t)\right) \mathrm{d} x+\int_{0}^{T} \int_{Q_{s}} \mathrm{D}\left(\frac{\partial \mathbf{u}^{s}}{\partial t}\right): \mathrm{D}\left(\frac{\partial \mathbf{u}^{s}}{\partial t}\right) \mathrm{d} x \leqslant C_{1}(\varepsilon), \\
\max _{0<t<T} \int_{Q_{f}}\left|\mathbf{u}^{f}(\mathbf{x}, t)\right|^{2} \mathrm{~d} x+\max _{0<t<T} \int_{Q_{s}}\left|\frac{\partial \mathbf{u}^{s}}{\partial t}(\mathbf{x}, t)\right|^{2} \mathrm{~d} x \leqslant C_{2}(\varepsilon) .
\end{gathered}
$$

Proof. First of all, note that, due to the linearity of the problem, it suffices to find the corresponding a priori estimates.

To prove estimate (2.26), we multiply the Stokes equation for $\mathbf{u}^{f}$ by $\mathbf{u}^{f}$ and integrate by parts over domain $Q_{f}$, multiply the Lamé equation for $\mathbf{u}^{s}$ by $\partial \mathbf{u}^{s} / \partial t$, integrate by parts over domain $Q_{s}$, and sum the results.

To get estimate (2.27), we differentiate the Stokes equation for $\mathbf{u}^{f}$ and the Lamé equation for $\mathbf{u}^{s}$ with respect to time, multiply the first expression by $\mathbf{u}^{f}$ and integrate by parts over domain $Q_{f} \times\left(0, t_{0}\right)$, multiply the second expression by $\partial \mathbf{u}^{s} / \partial t$ and integrate by parts over domain $Q_{s} \times\left(0, t_{0}\right)$, and sum the results:

$$
\begin{aligned}
& \frac{\mu}{2} \int_{Q_{f}} \mathrm{D}\left(\mathbf{u}^{f}\left(\mathbf{x}, t_{0}\right)\right): \mathrm{D}\left(\mathbf{u}^{f}\left(\mathbf{x}, t_{0}\right)\right) \mathrm{d} x+\lambda \int_{0}^{t_{0}} \int_{Q_{s}} \mathrm{D}\left(\frac{\partial \mathbf{u}^{s}}{\partial t}(\mathbf{x}, t)\right): \mathrm{D}\left(\frac{\partial \mathbf{u}^{s}}{\partial t}(\mathbf{x}, t)\right) \mathrm{d} x \mathrm{~d} t \\
& \quad=\int_{0}^{t_{0}} \int_{Q_{f}} \frac{\partial \widetilde{\rho}^{\varepsilon}}{\partial t}(\mathbf{x}, t)\left(\mathbf{u}^{f}(\mathbf{x}, t) \cdot \mathbf{e}\right) \mathrm{d} x \mathrm{~d} t \equiv I .
\end{aligned}
$$

Thus,

$$
\begin{aligned}
& \int_{Q_{f}} \mathrm{D}\left(\mathbf{u}^{f}\left(\mathbf{x}, t_{0}\right)\right): \mathrm{D}\left(\mathbf{u}^{f}\left(\mathbf{x}, t_{0}\right)\right) \mathrm{d} x+\int_{0}^{t_{0}} \int_{Q_{s}} \mathrm{D}\left(\frac{\partial \mathbf{u}^{s}}{\partial t}(\mathbf{x}, t)\right): \mathrm{D}\left(\frac{\partial \mathbf{u}^{s}}{\partial t}(\mathbf{x}, t)\right) \mathrm{d} x \mathrm{~d} t \\
& \leqslant|I| \leqslant \int_{0}^{t_{0}} \int_{Q_{f}}\left|\mathbf{u}^{f}(\mathbf{x}, t)\right|^{2} \mathrm{~d} x \mathrm{~d} t+C_{0}(\varepsilon) .
\end{aligned}
$$

To estimate the right-hand side in $(2.30)$, we introduce a new function $u(\mathbf{x}, t)$ :

$$
\mathbf{u}(\mathbf{x}, t)= \begin{cases}\mathbf{u}^{f}(\mathbf{x}, t) & \text { for } \mathbf{x} \in Q_{f} \\ \frac{\partial \mathbf{u}^{s}}{\partial t}(\mathbf{x}, t) & \text { for } \mathbf{x} \in Q_{s}\end{cases}
$$


It easy to see that $\mathbf{u} \in W_{2}^{1}(Q), \mathbf{u}(\mathbf{x}, t)=0$ for $\mathbf{x} \in S^{0}$ (see the boundary condition in (2.23)), and

$$
\begin{aligned}
\int_{Q}|\mathbf{u}(\mathbf{x}, t)|^{2} \mathrm{~d} x= & \int_{Q_{f}}\left|\mathbf{u}^{f}(\mathbf{x}, t)\right|^{2} \mathrm{~d} x+\int\left|\frac{\partial \mathbf{u}^{s}}{\partial t}(\mathbf{x}, t)\right|^{2} \mathrm{~d} x, \\
\int_{Q} \mathrm{D}(\mathbf{u}(\mathbf{x}, t)): \mathrm{D}(\mathbf{u}(\mathbf{x}, t)) \mathrm{d} x= & \int_{Q_{f}} \mathrm{D}\left(\mathbf{u}^{f}(\mathbf{x}, t)\right): \mathrm{D}\left(\mathbf{u}^{f}(\mathbf{x}, t)\right) \mathrm{d} x \\
& +\int_{0}^{t_{0}} \int_{Q_{s}} \mathrm{D}\left(\frac{\partial \mathbf{u}^{s}}{\partial t}(\mathbf{x}, t)\right): \mathrm{D}\left(\frac{\partial \mathbf{u}^{s}}{\partial t}(\mathbf{x}, t)\right) \mathrm{d} x \mathrm{~d} t .
\end{aligned}
$$

Therefore we may apply the Friedrichs-Poincaré inequality [17]

$$
\int_{Q}|\mathbf{u}(\mathbf{x}, t)|^{2} \mathrm{~d} x \leqslant C \int_{Q} \mathrm{D}(\mathbf{u}(\mathbf{x}, t)): \mathrm{D}(\mathbf{u}(\mathbf{x}, t)) \mathrm{d} x
$$

which, together with (2.30) and (2.31), implies

$$
\int_{Q_{f}} \mathrm{D}\left(\mathbf{u}^{f}\left(\mathbf{x}, t_{0}\right)\right): \mathrm{D}\left(\mathbf{u}^{f}\left(\mathbf{x}, t_{0}\right)\right) \mathrm{d} x \leqslant C \int_{0}^{t_{0}} \int_{Q_{f}} \mathrm{D}\left(\mathbf{u}^{f}(\mathbf{x}, t)\right): \mathrm{D}\left(\mathbf{u}^{f}(\mathbf{x}, t)\right) \mathrm{d} x \mathrm{~d} t+C_{0}(\varepsilon) .
$$

In turn, if we put

$$
y(t)=\int_{0}^{t} \int_{Q_{f}} \mathrm{D}\left(\mathbf{u}^{f}(\mathbf{x}, \tau)\right): \mathrm{D}\left(\mathbf{u}^{f}(\mathbf{x}, \tau)\right) \mathrm{d} x \mathrm{~d} \tau
$$

then we arrive at the differential inequality

$$
\frac{\mathrm{d} y}{\mathrm{~d} t}(t) \leqslant C y(t)+C_{0}(\varepsilon), \quad y(0)=0 .
$$

This last equation results in

$$
\max _{0<t<T} y(t) \leqslant C_{1}(\varepsilon)
$$

and, consequently, estimate (2.27).

Estimate (2.28) follows from (2.32).

Lemma 2. Under the conditions of Theorem 3, problem (2.20) has a unique weak solution.

Proof. For the given solution $\mathbf{u}^{f}=\Phi_{1}(\tilde{\rho})$ of problem (2.24), we may solve the initial boundary-value problem (2.25) and find $\rho=\Phi_{2}\left(\mathbf{u}^{f}\right)=\Phi(\widetilde{\rho})$ such that

$$
\rho^{-} \leqslant \rho(\mathbf{x}, t) \leqslant \rho^{+}, \quad \rho \in C^{2,1}(\bar{G}) .
$$

The first estimate follows from the maximum principle and shows that $\Phi$ transforms $\mathcal{M}$ into itself, and the smoothness of $\rho$ follows from existence theorems for parabolic equations with smooth coefficients [9]. 
So, if we prove the continuity of the operator $\Phi$, then $\Phi$ would be completely continuous due to (2.33). Finally, the Schauder fixed point theorem [6] allows us to find a fixed point of the operator $\Phi$ and solve problem (2.20).

The continuity of the linear operator $\Phi_{1}$

$$
\Phi_{1}: \mathcal{M} \rightarrow \mathcal{B}=L_{\infty}\left((0, T) ; W_{2}^{1}\left(Q_{f}\right)\right)
$$

follows from estimates (2.25).

The nonlinear operator $\Phi_{2}$ is also continuous. Indeed, let $\mathbf{u}_{1}^{f}, \mathbf{u}_{2}^{f} \in L_{\infty}\left((0, T) ; W_{2}^{1}\left(Q_{f}\right)\right)$. Then

$$
\mathbf{v}_{i}^{\epsilon}=\mathbf{M}_{\varepsilon}^{(1)}\left(\mathbf{M}_{\varepsilon}^{(2)}\left(\mathbf{u}_{i}^{f}\right)\right) \in C^{\infty}(\bar{G})
$$

and for differences

$$
\rho=\rho_{1}-\rho_{2}, \quad \rho_{i}=\Phi_{2}\left(\mathbf{u}_{i}^{f}\right), \quad \mathbf{v}=\mathbf{v}_{1}^{\varepsilon}-\mathbf{v}_{2}^{\varepsilon},
$$

we have

$$
\begin{aligned}
& \frac{\partial \rho}{\partial t}+\mathbf{v}_{1}^{\varepsilon} \cdot \nabla \rho=\mathbf{v} \cdot \nabla \rho_{2}, \quad \mathbf{x} \in Q, 0<t<T, \\
& \rho(\mathbf{x}, t)=0, \quad \mathbf{x} \in S_{f}^{ \pm}, 0<t<T, \quad \rho(\mathbf{x}, 0)=0, \quad \mathbf{x} \in Q .
\end{aligned}
$$

Therefore

$$
\left|\rho_{1}-\rho_{2}\right|_{G}^{(0)}=|\rho|_{G}^{(0)} \leqslant C_{1}(\varepsilon)|\mathbf{v}|_{G}^{(1)} \leqslant C_{2}(\varepsilon)\left\|\mathbf{u}_{1}^{f}-\mathbf{u}_{2}^{f}\right\|_{\mathcal{B}}
$$

which proves the complete continuity of the operator $\Phi$ and the solvability of problem (2.20).

To prove the uniqueness, we suppose that there exist two different solutions $\left(\mathbf{u}_{i}^{f, \varepsilon}, \mathbf{u}_{i}^{s, \varepsilon}, \rho_{i}^{\varepsilon}\right), i=1,2$, of problem (2.20).

The difference $\left\{\mathbf{u}^{f}, \mathbf{u}^{s}, \rho\right\}$,

$$
\mathbf{u}^{f}=\mathbf{u}_{1}^{f, \varepsilon}-\mathbf{u}_{2}^{f, \varepsilon}, \quad \mathbf{u}^{s}=\mathbf{u}_{1}^{\varepsilon, \varepsilon}-\mathbf{u}_{2}^{s, \varepsilon}, \quad \rho=\rho_{1}^{\varepsilon}-\rho_{2}^{\varepsilon},
$$

satisfies the following initial boundary-value problem:

$$
\begin{aligned}
& \nabla \cdot \mathrm{P}_{f}+\rho \mathbf{e}=0, \quad \nabla \cdot \mathbf{u}^{f}=0, \quad \mathbf{x} \in Q_{f}, 0<t<T, \\
& \nabla \cdot \mathrm{P}_{s}=0, \quad \nabla \cdot \mathbf{u}^{s}=0, \quad \mathbf{x} \in Q_{s}, 0<t<T, \quad \mathbf{u}^{s}(\mathbf{x}, 0)=0, \quad \mathbf{x} \in S, \\
& \mathbf{u}^{f}=\frac{\partial \mathbf{u}^{s}}{\partial t}, \quad \mathrm{P}_{f} \cdot \mathbf{n}=\mathrm{P}_{s} \cdot \mathbf{n}, \quad \mathbf{x} \in S, 0<t<T, \\
& \mathrm{P}_{i} \cdot \mathbf{e}_{1}=0, \quad \mathbf{x} \in S_{i}^{ \pm}, i=f, s, \quad \mathbf{u}^{s}(\mathbf{x}, t)=0, \quad \mathbf{x} \in S^{0}, 0<t<T, \\
& \frac{\partial \rho}{\partial t}+\mathbf{v}_{1}^{\varepsilon} \cdot \nabla \rho=\mathbf{v}^{\varepsilon} \cdot \nabla \rho_{2}^{\varepsilon}, \quad \mathbf{x} \in Q, 0<t<T, \\
& \rho(\mathbf{x}, t)=0, \quad \mathbf{x} \in S_{f}^{ \pm}, 0<t<T, \quad \rho(\mathbf{x}, 0)=0, \quad \mathbf{x} \in Q, \\
& \mathbf{v}^{\varepsilon}=\mathbf{M}_{\varepsilon}^{(1)}\left(\mathbf{M}_{\varepsilon}^{(2)}(\mathbf{u})\right), \quad \mathrm{P}_{f}=2 \mu \mathrm{D}\left(\mathbf{u}^{f}\right)-p_{f} \mathrm{I}, \quad \mathrm{P}_{s}=2 \lambda \mathrm{D}\left(\mathbf{u}^{s}\right)-p_{s} \mathrm{I} .
\end{aligned}
$$

Now we multiply the dynamic equation for $\mathbf{u}^{f}$ by $\mathbf{u}^{f}$ and integrate by parts over domain $Q_{f} \times\left(0, t_{0}\right)$, the dynamic equation for $\mathbf{u}^{s}$ by $\partial \mathbf{u}^{s} / \partial t$ and integrate by parts over domain $Q_{s} \times\left(0, t_{0}\right)$, the equation for $\rho$ by $\rho$ 
and integrate by parts over domain $Q_{f} \times\left(0, t_{0}\right)$, and finally sum all results:

$$
\begin{aligned}
& \mu \int_{0}^{t_{0}} \int_{Q_{f}} \mathrm{D}\left(\mathbf{u}^{f}(\mathbf{x}, t)\right): \mathrm{D}\left(\mathbf{u}^{f}(\mathbf{x}, t)\right) \mathrm{d} x \mathrm{~d} t+\frac{1}{2} \int_{Q_{f}}\left|\rho\left(\mathbf{x}, t_{0}\right)\right|^{2} \mathrm{~d} x \\
& \quad+\frac{\lambda}{2} \int_{Q_{s}} \mathrm{D}\left(\mathbf{u}^{s}\left(\mathbf{x}, t_{0}\right)\right): \mathrm{D}\left(\mathbf{u}^{s}\left(\mathbf{x}, t_{0}\right)\right) \mathrm{d} x \\
& =\int_{0}^{t_{0}} \int_{Q_{f}} \rho(\mathbf{x}, t)\left(\mathbf{u}^{f}(\mathbf{x}, t) \mathbf{e}\right) \mathrm{d} x \mathrm{~d} t \equiv I_{0} .
\end{aligned}
$$

Introducing the new function

$$
\mathbf{w}(\mathbf{x}, t)=\left\{\begin{array}{ll}
\int_{0}^{t} \mathbf{u}^{f}(\mathbf{x}, \tau) \mathrm{d} \tau, & \mathbf{x} \in Q_{f}, \\
\mathbf{u}^{s}(\mathbf{x}, t), & \mathbf{x} \in Q_{s},
\end{array} \quad \mathbf{u}(\mathbf{x}, t)=0, \quad \mathbf{x} \in S^{0},\right.
$$

in the same way as before (see estimates (2.26)-(2.28)), we get

$$
\left|I_{0}\right| \leqslant \delta \int_{0}^{t_{0}} \int_{Q_{f}}\left|\mathbf{u}^{f}(\mathbf{x}, t)\right|^{2} \mathrm{~d} x \mathrm{~d} t+C(\delta) \int_{0}^{t_{0}} \int_{Q_{f}}|\rho(\mathbf{x}, t)|^{2} \mathrm{~d} x \mathrm{~d} t
$$

for arbitrary small $\delta>0$ and

$$
\int_{Q_{f}}\left|\rho\left(\mathbf{x}, t_{0}\right)\right|^{2} \mathrm{~d} x \leqslant C(\delta) \int_{0}^{t_{0}} \int_{Q_{f}}|\rho(\mathbf{x}, t)|^{2} \mathrm{~d} x \mathrm{~d} t, \quad \int_{Q_{f}}|\rho(\mathbf{x}, 0)|^{2} \mathrm{~d} x=0 .
$$

The Gronwall inequality results in $\rho(\mathbf{x}, t)=0$ almost everywhere in $G$.

\subsubsection{Uniform bounds for the velocity and pressure of fluid}

Lemma 3. Under the conditions of Theorem 3 for the solution $\mathbf{u}^{\varepsilon}$ of problem (2.20), we have the following estimates:

$$
\begin{gathered}
\int_{0}^{T} \int_{Q_{f}} \mathrm{D}\left(\mathbf{u}^{f, \varepsilon}\right): \mathrm{D}\left(\mathbf{u}^{f, \varepsilon}\right) \mathrm{d} x \mathrm{~d} t+\max _{0<t<T} \int_{Q_{s}} \mathrm{D}\left(\mathbf{u}^{s, \varepsilon}(\mathbf{x}, t)\right): \mathrm{D}\left(\mathbf{u}^{s, \varepsilon}(\mathbf{x}, t)\right) \mathrm{d} x \leqslant C, \\
\max _{0<t<T} \int_{Q_{f}} \mathrm{D}\left(\mathbf{u}^{f, \varepsilon}(\mathbf{x}, t)\right): \mathrm{D}\left(\mathbf{u}^{f, \varepsilon}(\mathbf{x}, t)\right) \mathrm{d} x+\int_{0}^{T} \int_{Q_{s}} \mathrm{D}\left(\frac{\partial \mathbf{u}^{s, \varepsilon}}{\partial t}\right): \mathrm{D}\left(\frac{\partial \mathbf{u}^{s, \varepsilon}}{\partial t}\right) \mathrm{d} x \leqslant C, \\
\max _{0<t<T} \int_{Q_{f}}\left|\mathbf{u}^{f, \varepsilon}(\mathbf{x}, t)\right|^{2} \mathrm{~d} x+\max _{0<t<T} \int_{Q_{s}}\left|\frac{\partial \mathbf{u}^{s, \varepsilon}}{\partial t}(\mathbf{x}, t)\right|^{2} \mathrm{~d} x \leqslant C .
\end{gathered}
$$


Proof. The proof of these estimates almost completely repeats the proof of estimates (2.26)-(2.28). The only difference is in the estimation of the term $I$ in (2.29):

$$
\begin{aligned}
& \frac{\mu}{2} \int_{Q_{f}} \mathrm{D}\left(\mathbf{u}^{f, \varepsilon}\left(\mathbf{x}, t_{0}\right)\right): \mathrm{D}\left(\mathbf{u}^{f, \varepsilon}\left(\mathbf{x}, t_{0}\right)\right) \mathrm{d} x+\lambda \int_{0}^{t_{y}} \int_{Q_{s}} \mathrm{D}\left(\frac{\partial \mathbf{u}^{s, \varepsilon}}{\partial t}(\mathbf{x}, t)\right): \mathrm{D}\left(\frac{\partial \mathbf{u}^{s, \varepsilon}}{\partial t}(\mathbf{x}, t)\right) \mathrm{d} x \mathrm{~d} t \\
& =\int_{0}^{t_{0}} \int_{Q_{f}} \frac{\partial \rho^{\varepsilon}}{\partial t}(\mathbf{x}, t)\left(\mathbf{u}^{f, \varepsilon}(\mathbf{x}, t) \cdot \mathbf{e}\right) \mathrm{d} x \mathrm{~d} t \equiv I^{\varepsilon} .
\end{aligned}
$$

To estimate $I^{\varepsilon}$, we use the differential equation for $\rho^{\varepsilon}$ in $(2.21)$ :

$$
\begin{aligned}
\left|I^{\varepsilon}\right| & =\left|\int_{0}^{t_{0}} \int_{Q_{f}}\left(\mathbf{u}^{f, \varepsilon} \cdot \mathbf{e}\right) \nabla \cdot\left(\rho^{\varepsilon} \mathbf{v}^{\varepsilon}\right) \mathrm{d} x \mathrm{~d} t\right|=\left|\int_{0}^{t_{0}} \int_{Q_{f}}\left(\rho^{\varepsilon} \mathbf{v}^{\varepsilon}\right) \cdot \nabla \mathbf{u}^{f, \varepsilon} \cdot \mathbf{e} \mathrm{d} x \mathrm{~d} t\right| \\
& \leqslant \int_{0}^{t_{0}} \int_{Q_{f}} \mathrm{D}\left(\mathbf{u}^{f, \varepsilon}(\mathbf{x}, t)\right): \mathrm{D}\left(\mathbf{u}^{f, \varepsilon}(\mathbf{x}, t)\right) \mathrm{d} x \mathrm{~d} t+C \int_{0}^{t_{0}} \int_{Q_{f}}\left(\mathbf{u}^{f, \varepsilon}(\mathbf{x}, t)\right)^{2} \mathrm{~d} x \mathrm{~d} t+C,
\end{aligned}
$$

where we have used the evident estimates for mollifiers $\mathbf{M}_{\varepsilon}^{(1)}\left(\mathbf{M}_{\varepsilon}^{(2)}\right)$,

$$
\int_{0}^{t_{0}} \int_{Q_{f}}\left(\mathbf{v}^{\varepsilon}(\mathbf{x}, t)\right)^{2} \mathrm{~d} x \mathrm{~d} t \leqslant C \int_{0}^{t_{0}} \int_{Q_{f}}\left(\mathbf{u}^{f, \varepsilon}(\mathbf{x}, t)\right)^{2} \mathrm{~d} x \mathrm{~d} t .
$$

The rest of the proof is the same as that for (2.26)-(2.28).

Lemma 4. Under the conditions of Theorem 3 , let $\mathbf{u}^{f, \varepsilon}$ be the solution of problem (2.20). Then $\mathrm{P}_{f}\left(\mathbf{u}^{f, \varepsilon}, p_{f}^{\varepsilon}\right) \in$ $L_{\infty}\left((0, T) ; L_{2}\left(Q_{f}\right)\right)$,

$$
\max _{0<t<T} \int_{Q_{f}}\left|p_{f}^{\varepsilon}(\mathbf{x}, t)\right|^{2} \mathrm{~d} x \leqslant C
$$

$\mathrm{P}_{s}\left(\mathbf{u}^{s, \varepsilon}, p_{s}^{\varepsilon}\right) \in L_{\infty}\left((0, T) ; L_{2}\left(Q_{s}\right)\right)$,

$$
\max _{0<t<T} \int_{Q_{s}}\left|p_{s}^{\varepsilon}(\mathbf{x}, t)\right|^{2} \mathrm{~d} x \leqslant C,
$$

and for any $\Omega_{f} \subset Q_{f}$ and $\Omega_{s} \subset Q_{s}$,

$$
\mathbf{u}^{f, \varepsilon} \in L_{\infty}\left((0, T) ; W_{m}^{2}\left(\Omega_{f}\right)\right), \quad \mathbf{u}^{s, \varepsilon} \in L_{\infty}\left((0, T) ; W_{m}^{2}\left(\Omega_{s}\right)\right)
$$

for all any $m>2$.

Proof. Let $\varphi \in \stackrel{\circ}{W}_{2}^{1} Q_{f}$ ) be a test function in the integral identity (2.22). Then this identity takes the form

$$
\int_{Q_{f}} p_{f}^{\varepsilon} \nabla \cdot \varphi \mathrm{d} x=\int_{Q_{f}}\left(2 \mu \mathrm{D}\left(\mathbf{u}^{f, \varepsilon}\right): \mathrm{D}(\varphi)-\left(\mathbf{f}+\rho^{\varepsilon} \mathbf{e}\right) \cdot \varphi\right) \mathrm{d} x .
$$


Now we choose $\varphi$ as a solution of the problem

$$
\begin{aligned}
& \varphi=\varphi_{0}+\nabla \psi, \quad \Delta \psi=p_{f}^{\varepsilon}, \quad \mathbf{x} \in Q_{f}, \quad \psi(\mathbf{x}, t)=0, \quad \mathbf{x} \in S, 0<t<T, \\
& \nabla \cdot \varphi_{0}=0, \quad \mathbf{x} \in Q_{f}, \quad \nabla \psi(\mathbf{x}, t)+\varphi_{0}(\mathbf{x}, t)=0, \quad \mathbf{x} \in S, 0<t<T .
\end{aligned}
$$

This problem has a unique solution [7], and

$$
\max _{0<t<T} \int_{Q_{f}}|\nabla \varphi(\mathbf{x}, t)|^{2} \mathrm{~d} x \leqslant C\left\|p_{f}^{\varepsilon}\right\|_{2, Q_{f}}^{2} .
$$

Thus (2.34)-(2.38) result in

$$
\max _{0<t<T} \int_{Q_{f}}\left|p_{f}^{\varepsilon}(\mathbf{x}, t)\right|^{2} \mathrm{~d} x \leqslant C,
$$

and $\mathrm{P}_{f}\left(\mathbf{u}^{f, \varepsilon}, p_{f}^{\varepsilon}\right) \in L_{\infty}\left((0, T) ; L_{2}\left(Q_{f}\right)\right)$.

Coming back to (2.37), we conclude that $\mathrm{P}_{f}\left(\mathbf{u}^{f, \varepsilon}, p_{f}^{\varepsilon}\right) \in L_{\infty}\left((0, T) ; W_{2}^{1}\left(Q_{f}\right)\right)$, and (2.37) is equivalent to the Stokes equation

$$
\mu \Delta \mathbf{u}^{f, \varepsilon}-\nabla p_{f}^{\varepsilon}+\mathbf{f}+\rho^{\varepsilon} \mathbf{e}=0, \quad \mathbf{x} \in Q_{f}, 0<t<T .
$$

The right-hand side $\mathbf{F}=\mathbf{f}+\rho^{\varepsilon}$ e of the differential equation belongs to $L_{\infty}(G)$. Therefore we may use the same arguments as in [12] and conclude that, for any $\Omega \subset Q_{f}$,

$$
\mathbf{u}^{f, \varepsilon} \in L_{\infty}\left((0, T) ; W_{m}^{2}(\Omega)\right) \text { for any } m>2
$$

We may apply the same arguments for the solid component:

$$
\max _{0<t<T} \int_{Q_{s}}\left|p_{s}^{\varepsilon}(\mathbf{x}, t)\right|^{2} \mathrm{~d} x \leqslant C,
$$

and $\mathrm{P}_{s}\left(\mathbf{u}^{s, \varepsilon}, p_{s}^{\varepsilon}\right) \in L_{\infty}\left((0, T) ; L_{2}\left(Q_{s}\right)\right)$.

Lemma 5. Under the conditions of Theorem 3 , let $\mathbf{u}^{f, \varepsilon}$ be the solution of problem (2.20). Then

$$
\max _{0<t<T}\left|\mathbf{u}^{f, \varepsilon}(\cdot, t)\right|_{Q_{f}^{(2 \delta)}}^{(1+\alpha)} \leqslant C(\alpha, \delta)
$$

for $0<\delta<\delta_{0}$ with sufficiently small $\delta_{0}$ and any $0<\alpha<1$.

Proof. The domain $Q_{f}^{(\delta)}$ consist of two disconnected parts. For each part, the proof is the same. Note also that

$$
\max _{0<t<T}\left|\mathbf{u}^{f, \varepsilon}(\cdot, t)\right|_{\Omega^{(f, \delta)}}^{(1+\alpha)} \leqslant C(\alpha, \delta)
$$

for any domain $\Omega^{(f, \delta)} \subset Q_{f}^{(\delta)}$ with the distance to the solid part $Q_{s}$ greater than $\delta$.

So, we may restrict ourselves only with the part in $x_{2}<0$ and domains $\Omega^{(\delta)}$. 
For $0<\delta<1 / 2$, we put

$$
\Omega^{(\delta)}=\left\{\mathrm{x} \in Q:-1+\delta<x_{1}<1-\delta,-\frac{1}{2}+\delta<x_{2}<-\frac{1}{2}-\delta\right\}, \quad \Omega_{f}^{(\delta)}=\Omega^{(\delta)} \cap Q_{f} .
$$

Let $\zeta^{(\delta)}(\mathbf{x})$ be infinitely smooth functions such that $\zeta^{(\delta)}(\mathbf{x})=1$ for $\mathbf{x} \in \Omega^{(2 \delta)}$ and $\zeta^{(\delta)}(\mathbf{x})=0$ for $\mathbf{x} \in Q \backslash \Omega^{(\delta)}$.

Then the functions $\mathbf{u}^{f, \varepsilon, \delta}=\mathbf{u}^{f, \varepsilon} \zeta^{(\delta)}, p_{f}^{\varepsilon, \delta}=p_{f}^{\varepsilon} \zeta^{(\delta)}, \mathbf{u}^{s, \varepsilon, \delta}=\mathbf{u}^{s, \varepsilon} \zeta^{(\delta)}$, and $p_{s}^{\varepsilon, \delta}=p_{s}^{\varepsilon} \zeta^{(\delta)}$ satisfy the following linear problem:

$$
\begin{aligned}
& \mu \Delta \mathbf{u}^{f, \varepsilon, \delta}-\nabla p_{f}^{\varepsilon, \delta}=\mathbf{F}^{\varepsilon, \delta}, \quad \nabla \cdot \mathbf{u}^{f, \varepsilon, \delta}=\varphi^{\varepsilon, \delta}, \quad x_{2}>-\frac{1}{2}, 0<t<T ; \\
& \lambda \Delta \mathbf{u}^{s, \varepsilon, \delta}-\nabla p_{s}^{\varepsilon, \delta}=\mathbf{F}^{\varepsilon, \delta}, \quad \nabla \cdot \mathbf{u}^{s, \varepsilon, \delta}=\varphi^{\varepsilon, \delta}, \quad x_{2}<-\frac{1}{2}, 0<t<T ; \\
& \mu\left(\frac{\partial}{\partial x_{2}} u_{1}^{f, \varepsilon, \delta}+\frac{\partial}{\partial x_{1}} u_{2}^{f, \varepsilon, \delta}\right)=\lambda\left(\frac{\partial}{\partial x_{2}} u_{1}^{s, \varepsilon, \delta}+\frac{\partial}{\partial x_{1}} u_{2}^{s, \varepsilon, \delta}\right)+\psi, \\
& \mu \frac{\partial}{\partial x_{2}} u_{2}^{f, \varepsilon, \delta}+p_{f}^{\varepsilon, \delta}=\lambda \frac{\partial}{\partial x_{2}} u_{2}^{s, \varepsilon, \delta}+p_{s}^{\varepsilon, \delta}, \\
& u_{1}^{f, \varepsilon, \delta}=\frac{\partial}{\partial t} u_{1}^{s, \varepsilon, \delta}, \quad u_{2}^{f, \varepsilon, \delta}=\frac{\partial}{\partial t} u_{2}^{s, \varepsilon, \delta}, \quad x_{2}=\frac{1}{2}, 0<t<T ; \\
& \mathbf{u}^{s, \varepsilon, \delta}(\mathbf{x}, 0)=0, \quad x_{2}=-\frac{1}{2} .
\end{aligned}
$$

Here

$$
\begin{aligned}
& \mathbf{F}^{\varepsilon, \delta}=\zeta^{(\delta)}\left(\rho^{\varepsilon} \mathbf{e}+\mathbf{f}\right)+\mu \nabla \mathbf{u}^{f, \varepsilon, \delta} \cdot \nabla \zeta^{(\delta)}+p_{f}^{\varepsilon, \delta} \nabla \zeta^{(\delta)}, \\
& \varphi^{\varepsilon, \delta}=\mathbf{u}^{f, \varepsilon, \delta} \cdot \nabla \zeta^{(\delta)}, \quad x_{2}>-\frac{1}{2} ; \quad \psi=\frac{\partial \zeta^{(\delta)}}{\partial x_{1}}\left(\mu u_{2}^{f, \varepsilon, \delta}-\lambda u_{2}^{s, \varepsilon, \delta}\right), \quad x_{2}=-\frac{1}{2} ; \\
& \mathbf{F}^{\varepsilon, \delta}=\zeta^{(\delta)} \mathbf{f}+\lambda \nabla \mathbf{u}^{s, \varepsilon, \delta} \cdot \nabla \zeta^{(\delta)}+p_{s}^{\varepsilon, \delta} \nabla \zeta^{(\delta)}, \quad \varphi^{\varepsilon, \delta}=\mathbf{u}^{s, \varepsilon, \delta} \cdot \nabla \zeta^{(\delta)}, \quad x_{2}<-\frac{1}{2} ; \\
& \max _{0<t<T}\left\|\mathbf{F}^{\varepsilon, \delta}(., t)\right\|_{2, \mathbb{R}^{2}}+\max _{0<t<T}\left\|\varphi^{\varepsilon, \delta}(., t)\right\|_{2, \mathbb{R}^{2}}^{(1)} \leqslant C(\delta) .
\end{aligned}
$$

For simplicity, we denote all constants independent of $\varepsilon$ as $C$ (or $C(\delta)$ ) and omit for the moment the indices $\varepsilon$ and $\delta$.

Now we reduce (2.40) to homogeneous differential equations by introducing new functions $\left\{\mathbf{w}^{f}, r_{f}, \mathbf{w}^{s}, r_{s}\right\}$ as a solution to the following problem:

$$
\begin{aligned}
& \mu \Delta \mathbf{w}^{f}-\nabla r_{f}=\mathbf{F}, \quad \nabla \cdot \mathbf{w}^{f}=\varphi, \quad x_{2}+\frac{1}{2}>0,0<t<T ; \\
& \lambda \Delta \mathbf{w}^{s}-\nabla r_{s}=\mathbf{F}, \quad \nabla \cdot \mathbf{w}^{s}=\varphi, \quad x_{2}+\frac{1}{2}<0,0<t<T ; \\
& \mu\left(\frac{\partial w_{1}^{f}}{\partial x_{2}}+\frac{\partial w_{2}^{f}}{\partial x_{1}}\right)=\lambda\left(\frac{\partial w_{1}^{s}}{\partial x_{2}}+\frac{\partial w_{2}^{s}}{\partial x_{1}}\right)=\psi, \\
& \mu \frac{\partial w_{2}^{f}}{\partial x_{2}}+r_{f}=\lambda \frac{\partial w_{2}^{s}}{\partial x_{2}}+r_{s}=0, \quad x_{2}=-\frac{1}{2}, 0<t<T .
\end{aligned}
$$


Thus, for

$$
\mathbf{v}=\mathbf{u}-\mathbf{w}, \quad q=p-r,
$$

we have

$$
\begin{aligned}
& \mu \Delta \mathbf{v}^{f}-\nabla q_{f}=0, \quad \nabla \cdot \mathbf{v}^{f}=0, \quad x_{2}+\frac{1}{2}>0,0<t<T ; \\
& \lambda \Delta \mathbf{v}^{s}-\nabla q_{s}=0, \quad \nabla \cdot \mathbf{v}^{s}=0, \quad x_{2}+\frac{1}{2}<0,0<t<T ; \\
& \mu\left(\frac{\partial v_{1}^{f}}{\partial x_{2}}+\frac{\partial v_{2}^{f}}{\partial x_{1}}\right)=\lambda\left(\frac{\partial v_{1}^{s}}{\partial x_{2}}+\frac{\partial v_{2}^{s}}{\partial x_{1}}\right), \\
& \mu \frac{\partial v_{2}^{f}}{\partial x_{2}}+q_{f}=\lambda \frac{\partial v_{2}^{s}}{\partial x_{2}}+q_{s}, \\
& \frac{\partial v_{1}^{s}}{\partial t}=v_{1}^{f}+\varphi_{1}, \quad \frac{\partial v_{2}^{s}}{\partial t}=v_{2}^{f}+\varphi_{2}, \quad x_{2}=-\frac{1}{2}, 0<t<T,
\end{aligned}
$$

where

$$
\varphi\left(x_{1}, t\right)=\left(\varphi_{1}\left(x_{1}, t\right), \varphi_{2}\left(x_{1}, t\right)\right)=\mathbf{w}^{f}\left(x_{1}, 0, t\right)-\frac{\partial \mathbf{w}^{s}}{\partial t}\left(x_{1}, 0, t\right) .
$$

Note that, due to the homogeneous boundary condition in (2.20) for $\mathbf{u}^{s}$ at $S$, we have $\partial \mathbf{u}^{s} / \partial t(\mathbf{x}, 0) \equiv 0$ in $Q_{s}$, which implies

$$
\frac{\partial \mathbf{w}^{s}}{\partial t}(\mathbf{x}, 0) \equiv 0 \quad \text { and } \quad \varphi_{i}\left(x_{1}, 0\right)=w_{i}^{f}\left(x_{1}, 0\right) \quad \text { for } i=1,2 .
$$

To solve (2.43), we apply the Fourier transformation

$$
\widehat{v}\left(\xi, x_{2}, t\right)=\frac{1}{\sqrt{2 \pi}} \int_{-\infty}^{\infty} v\left(x_{1}, x_{2}, t\right) \mathrm{e}^{-\mathrm{i} \xi x_{1}} \mathrm{~d} x_{1}
$$

with respect the variable $x_{1}$ and get the following system of ordinary differential equations in the variable $x_{2}$ :

$$
\begin{aligned}
& \mu \frac{\partial^{2} \widehat{v}_{1}^{f}}{\partial x^{2}}-\mu \xi^{2} \widehat{v}_{1}^{f}+\mathrm{i} \xi \widehat{p}_{f}=0, \quad \mu \frac{\partial^{2} \widehat{v}_{2}^{f}}{\partial x^{2}}-\mu \xi^{2} \widehat{v}_{2}^{f}-\frac{\partial \widehat{p}_{f}}{\partial x_{2}}=0, \\
& \frac{\partial^{2} \widehat{p}_{f}}{\partial x^{2}}-\xi^{2} \widehat{p}_{f}=0, \quad \widehat{v}_{1}^{f}=-\frac{\mathrm{i}}{\xi} \frac{\partial \widehat{v}_{2}^{f}}{\partial x_{2}}, \quad x_{2}+\frac{1}{2}>0 \\
& \lambda \frac{\partial^{2} \widehat{v}_{1}^{s}}{\partial x^{2}}-\lambda \xi^{2} \widehat{v}_{1}^{s}+\mathrm{i} \xi \widehat{p}_{f}=0, \quad \lambda \frac{\partial^{2} \widehat{v}_{2}^{s}}{\partial x^{2}}-\lambda \xi^{2} \widehat{v}_{2}^{s}-\frac{\partial \widehat{p}_{s}}{\partial x_{2}}=0, \\
& \frac{\partial^{2} \widehat{p}_{s}}{\partial x^{2}}-\xi^{2} \widehat{p}_{s}=0, \quad \widehat{v}_{1}^{s}=-\frac{i}{\xi} \frac{\partial \widehat{v}_{2}^{s}}{\partial x_{2}}, \quad x_{2}+\frac{1}{2}<0 \\
& \mu \frac{\partial \hat{v}_{1}^{f}}{\partial x_{2}}-\mu \mathrm{i} \xi \widehat{v}_{2}^{f}=\lambda \frac{\partial \widehat{v}_{1}^{s}}{\partial x_{2}}-\lambda \mathrm{i} \xi \widehat{v}_{2}^{s}, \quad \mu \frac{\partial \widehat{v}_{2}^{f}}{\partial x_{2}}+\widehat{p}_{f}=\lambda \frac{\partial \widehat{v}_{2}^{s}}{\partial x_{2}}+\widehat{p}_{s}, \\
& \widehat{v}_{1}^{f}+\widehat{\varphi}_{1}=\frac{\partial \widehat{v}_{1}^{s}}{\partial t}, \quad \widehat{v}_{2}^{f}+\widehat{\varphi}_{2}=\frac{\partial \widehat{v}_{2}^{s}}{\partial t}, \quad x_{2}=-\frac{1}{2} ; \\
& \widehat{v}_{1}^{s}\left(\xi, x_{2}, 0\right)=\widehat{v}_{2}^{s}\left(\xi, x_{2}, 0\right)=0 .
\end{aligned}
$$


Solutions of (2.44) and (2.45) have a very simple form

$$
\widehat{p}_{f}=c_{p}^{f} \mathrm{e}^{-|\xi| z}, \quad \widehat{v}_{1}^{f}=\mathrm{i}\left(\frac{|\xi|}{\xi} c_{v}^{f}-\frac{1}{2 \mu \xi}(1-z|\xi|) c_{p}^{f}\right) \mathrm{e}^{-|\xi| z}, \quad \widehat{v}_{2}^{f}=\left(c_{n}^{f}+\frac{z}{2 \mu} c_{p}^{f}\right) \mathrm{e}^{-|\xi| z}
$$

where $z=\left|x_{2}+1 / 2\right|$,

$$
\widehat{p}_{s}=c_{p}^{s} \mathrm{e}^{|\xi| z}, \quad \widehat{v}_{1}^{s}=-\mathrm{i}\left(\frac{|\xi|}{\xi} c_{v}^{s}+\frac{1}{2 \lambda \xi}(1+z|\xi|) c_{p}^{s}\right) \mathrm{e}^{|\xi| z}, \quad \widehat{v}_{2}^{s}=\left(c_{v}^{f}+\frac{z}{2 \mu} c_{p}^{f}\right) \mathrm{e}^{-|\xi| z} .
$$

To define the functions $c_{p}^{f}, c_{v}^{f}, c_{p}^{s}$, and $c_{v}^{s}$, we use boundary conditions (2.46):

$$
\begin{aligned}
& c_{v}^{s}+\frac{1}{2 \lambda|\xi|} c_{p}^{s}=\frac{\mu}{\lambda} c_{v}^{f}-\frac{1}{2 \lambda|\xi|} c_{p}^{f}, \quad c_{v}^{s}+\frac{3}{2 \lambda|\xi|} c_{p}^{s}=-\frac{\mu}{\lambda} c_{v}^{f}+\frac{3}{2 \lambda|\xi|} c_{p}^{f}, \\
& \frac{\partial c_{v}^{s}}{\partial t}+\frac{1}{2 \lambda|\xi|} \frac{\partial c_{p}^{s}}{\partial t}=-c_{v}^{f}+\frac{1}{2 \mu|\xi|} c_{p}^{f}+\mathrm{i} \frac{|\xi|}{\xi} \widehat{\varphi}_{1}, \quad \frac{\partial c_{v}^{s}}{\partial t}=c_{v}^{f}+\widehat{\varphi}_{2}, \\
& c_{v}^{s}(\xi, 0)=c_{p}^{s}(\xi, 0)=0 .
\end{aligned}
$$

The first system (2.47) gives us $c_{v}^{s}$ and $c_{p}^{s}$ as combinations of $c_{v}^{f}$ and $c_{p}^{f}$ :

$$
c_{v}^{s}=2 \frac{\mu}{\lambda} c_{v}^{f}-\frac{3}{2 \lambda|\xi|} c_{p}^{f}, \quad c_{p}^{s}=-2 \mu|\xi| c_{v}^{f}+2 c_{p}^{f}
$$

Taking into account (2.48) and (2.49), we define $c_{v}^{-}$and $c_{r}^{-}$from the Cauchy problem for the following system of ordinary differential equations:

$$
\begin{aligned}
& \frac{\partial c_{v}^{f}}{\partial t}=-4 \frac{\lambda}{\mu} c_{v}^{f}+\frac{3 \lambda}{4 \mu^{2}|\xi|} c_{p}^{f}+3 \mathrm{i} \frac{\lambda|\xi|}{\mu \xi} \widehat{\varphi}_{1}-\frac{\lambda}{\mu} \widehat{\varphi}_{2}, \\
& \frac{\partial c_{p}^{f}}{\partial t}=-6 \lambda|\xi| c_{v}^{f}+2 \frac{\lambda}{\mu} c_{p}^{f}+4 \mathrm{i} \xi \widehat{\varphi}_{1}-2 \lambda|\xi| \widehat{\varphi}_{2}, \\
& c_{v}^{f}(\xi, 0)=c_{p}^{f}(\xi, 0)=0 .
\end{aligned}
$$

The last equation is equivalent to the Cauchy problem for the second-order ordinary differential equation with coefficient $k^{2}=32 \lambda / \mu$ independent of $\xi$ :

$$
\begin{aligned}
& \frac{\partial^{2} c_{v}^{f}}{\partial t^{2}}-k^{2} c_{v}^{f}=3 \frac{\lambda}{\mu} \widehat{\varphi}_{2}+\frac{\partial \widehat{\varphi}_{1}}{\partial t}-4 \frac{\lambda}{\mu} \widehat{\varphi}_{1}, \\
& c_{v}^{f}(\xi, 0)=0, \quad \frac{\partial c_{v}^{f}}{\partial t}(\xi, 0)=3 \mathrm{i} \frac{\lambda|\xi|}{\mu \xi} \widehat{\varphi}_{1}(\xi, 0)-\frac{\lambda}{\mu} \widehat{\varphi}_{2}(\xi, 0) .
\end{aligned}
$$

Thus, for $j=f, s$ and $l=v, p$,

$$
c_{l}^{j}(\xi, t)=\sum_{m=f, s} \sum_{i=1}^{2}\left(Z_{l, i}^{j, m}(t) \hat{w}_{i}^{m}(\xi, 0,0)+\int_{0}^{t} G_{l, i}^{j, m}(t-\tau) \widehat{w}_{i}^{m}(\xi, 0, \tau) \mathrm{d} \tau\right) .
$$

Note that $\widehat{w}_{i}^{k}(\xi, 0,0), z_{l}^{j, i}$, and $g_{l}^{j, i}$ are known functions and that $z_{l}^{j, i}$ and $g_{l}^{j, i}$ are infinitely smooth in $t$. 
Gathering all these together, we have, for $j=f, s$ and $l=1,2$,

$$
\begin{aligned}
\widehat{v}_{l}^{j}(\xi, z, t)= & \sum_{m=f, s} \sum_{i=1}^{2}\left\{\left(\left(Z_{l, i, 0}^{j, m}(z, t)+\frac{|\xi|}{\xi} Z_{l, i, 1}^{j, m}(z, t)\right) \widehat{w}_{i}^{m}(\xi, 0,0)\right) \mathrm{e}^{-|\xi| z}\right. \\
& \left.+\left(\int_{0}^{t}\left(G_{l, i, 0}^{j, m}(z, t-\tau)+\frac{|\xi|}{\xi} G_{l, i, 1}^{j, m}(z, t-\tau)\right) \widehat{w}_{i}^{m}(\xi, 0, \tau) \mathrm{d} \tau\right)\right\} \mathrm{e}^{-|\xi| z},
\end{aligned}
$$

where $Z_{l, i . k}^{j, m}(z, t)$ and $G_{l, i . k}^{j, m}(z, t), k=0,1$, are linear in $z \geqslant 0$ and infinitely smooth in $t$.

For $z=0$, we have

$$
\begin{aligned}
& \widehat{v}_{l}^{j}(\xi, 0, t)= \sum_{m=f, s} \sum_{i=1}^{2}\left\{\left(\left(Z_{l, i, 0}^{j, m}(0, t)+\frac{|\xi|}{\xi} Z_{l, i, 1}^{j, m}(0, t)\right) \widehat{w}_{i}^{m}(\xi, 0,0)\right)\right. \\
&\left.+\left(\int_{0}^{t}\left(G_{l, i, 0}^{j, m}(0, t-\tau)+\frac{|\xi|}{\xi} G_{l, i, 1}^{j, m}(0, t-\tau)\right) \widehat{w}_{i}^{m}(\xi, 0, \tau) \mathrm{d} \tau\right)\right\}, \\
& \max _{0<t<T}\left\|\mathbf{v}^{j}(., t)\right\|_{2, \mathbb{R}_{j}^{2}}^{(l)} \leqslant C_{1} \max _{0<t<T}\left\|\mathbf{v}^{j}(., t)\right\|_{2, \mathbb{R}^{k}}^{(l-1 / 2)} \leqslant C_{2} \max _{0<t<T}\left\|\mathbf{w}^{j}(., t)\right\|_{2, \mathbb{R}}^{(l-1 / 2)} \\
& \leqslant C_{3} \max _{0<t<T}\left\|\mathbf{w}^{j}(., t)\right\|_{2, \mathbb{R}_{j}^{2},}^{(l)}, j=f, s .
\end{aligned}
$$

Coming back to the previous notations and taking into account (2.41) and the definition of $\mathbf{v}^{j, \varepsilon, \delta}$ and $\mathbf{w}^{j, \varepsilon, \delta}$, from (2.50) we get, for $l=2$,

$$
\max _{0<t<T}\left\|\mathbf{u}^{j, \varepsilon}(., t)\right\|_{2, Q_{j}^{(\delta)}}^{(2)} \leqslant C(\delta), \quad j=f, s .
$$

Now we repeat with function $\zeta^{(2 \delta)}$ and domain $\Omega^{(2 \delta)}: \zeta^{(2 \delta)}(\mathbf{x})=1$ for $\mathbf{x} \in \Omega^{(4 \delta)}$ and $\zeta^{(2 \delta)}(\mathbf{x})=0$ for $\mathbf{x} \in Q \backslash \Omega^{(2 \delta)}$.

Namely, for (2.42), (2.51) implies

$$
\max _{0<t<T}\left\|\mathbf{F}^{\varepsilon, 2 \delta}(., t)\right\|_{2, \mathbb{R}^{2}}^{(1)}+\max _{0<t<T}\left\|\varphi^{\varepsilon, \delta}(., t)\right\|_{2, \mathbb{R}^{2}}^{(2)} \leqslant C(\delta),
$$

and, consequently,

$$
\begin{gathered}
\max _{0<t<T}\left\|\mathbf{v}^{j \varepsilon, 2 \delta}(., t)\right\|_{2, \mathbb{R}_{j}^{2}}^{(3)} \leqslant C_{1} \max _{0<t<T}\left\|\mathbf{w}^{j \varepsilon, 2 \delta}(., t)\right\|_{2, \mathbb{R}_{j}^{2}}^{(3)} \leqslant C_{2}(\delta), \quad j=f, s, \\
\max _{0<t<T}\left\|\mathbf{u}^{j, \varepsilon}(., t)\right\|_{2, Q_{j}^{(2 \delta)}}^{(3)} \leqslant C_{3}(\delta), \quad j=f, s .
\end{gathered}
$$

The corresponding imbedding theorem $W_{2}^{3}(\Omega) \rightarrow C^{1+\alpha}(\Omega)$ for $0<\alpha<1$ [9],

$$
\max _{0<t<T}\left|\mathbf{u}^{j, \varepsilon}(., t)\right|_{Q_{j}^{(2 \delta)}}^{(1+\alpha)} \leqslant C \max _{0<t<T}\left\|\mathbf{u}^{j, \varepsilon}(., t)\right\|_{2, Q_{j}^{(2 \delta)}}^{(3)} \leqslant C_{4}(\delta), \quad j=f, s,
$$

proves (2.39) and the statement of the lemma. 


\subsubsection{Uniform bounds for density}

Let $\Gamma^{\varepsilon}(t) \subset Q_{f}$ be a smooth surface obtained by moving the initial position $\Gamma(0)$ along the trajectories of the velocity field $\mathbf{v}^{\varepsilon}$ :

$$
\frac{\mathrm{d} \mathbf{x}}{\mathrm{d} t}=\mathbf{v}^{\varepsilon}(\mathbf{x}, t), \quad \mathbf{x}(0)=\xi, \quad \xi \in \Gamma(0)
$$

As we have mentioned previously, the time $T$ is chosen from the condition

$$
\operatorname{dist}\left(\Gamma^{\varepsilon}(t), S^{ \pm}\right)>0
$$

Moreover, we suppose that

$$
\operatorname{dist}\left(\Gamma^{\varepsilon}(t), S^{ \pm}\right)>\varepsilon
$$

Lemma 6. Under the conditions of Theorem 3, let $\rho^{\varepsilon}$ be the solution of problem (2.21). Then

$$
\max _{0<t<T}\left(\int_{Q_{f}^{(\delta)}}\left|\frac{\partial \rho^{\varepsilon}}{\partial t}(\mathbf{x}, t)\right| \mathrm{d} x+\sum_{i=1}^{2} \int_{Q_{f}^{(\delta)}}\left|\frac{\partial \rho^{\varepsilon}}{\partial x_{i}}(\mathbf{x}, t)\right| \mathrm{d} x\right) \leqslant C(\delta) .
$$

Proof. $\quad$ Let $q_{i}=\partial \rho^{\varepsilon} / \partial x_{i}$

Then

$$
\frac{\partial q_{i}}{\partial t}+\mathbf{v}^{\varepsilon} \cdot \nabla q_{i}=\sum_{j=1}^{n} a_{i j} q_{j}, \quad \mathbf{x} \in Q, 0<t<T,
$$

where $a_{i j}=-\partial v_{j}^{\varepsilon} / \partial x_{i}, i, j=1,2$, and

$$
\max _{0<t<T}\left|a_{i j}(., t)\right|_{2, Q_{j}^{(2 \delta)}}^{(1)} \leqslant C(\delta)
$$

Note that $q_{i} \equiv 0$ near the boundaries $S^{ \pm}$and $S^{0}$. This follows from the supposition on the behavior of the boundary $\Gamma^{\varepsilon}(t)$ and from the choice of the time $T$.

Multiplying the equation for $q_{i}$ by $q_{i} /\left(q_{i}^{2}+\delta^{2}\right)^{1 / 2}$ and integrating by parts over $Q$, we arrive at the equality

$$
\frac{\mathrm{d}}{\mathrm{d} t} \int_{Q}\left(q_{i}^{2}+\delta^{2}\right)^{1 / 2} \mathrm{~d} x=-\int_{Q} \sum_{j=1}^{2} a_{i j} q_{j} \frac{q_{i}}{\left(q_{i}^{2}+\delta^{2}\right)^{1 / 2}} \mathrm{~d} x
$$

and, consequently, the inequality

$$
\frac{\mathrm{d} y}{\mathrm{~d} t} \leqslant C(\delta) y, \quad y(0) \leqslant C(\delta)
$$

for $y=\sum_{i=1}^{2} \int_{Q}\left(q_{i}^{2}+\delta^{2}\right)^{1 / 2} \mathrm{~d} x$.

The Gronwall inequality provides estimates (2.52) for $q_{i}, i=1,2$, and the transport equation (2.21) provides estimate (2.52) for the time derivative of $\rho^{\varepsilon}$.

Passage to nonsmooth initial data, the existence of a regular free boundary, the existence of the maximal time interval, and the uniqueness of the solution are proved in the same way as in [1] and [12]. 


\section{References}

1. S. Antontsev, Meirmanov A., and V. Yurinsky, A free-boundary problem for Stokes equations: Classical solutions, Interfaces Free Bound., 2(4):413-424, 2000.

2. S.N. Antontsev, A.V. Kazhikhov, and V.N. Monakhov, Boundary Value Problems in Mechanics of Nonhomogeneous Fluids, North-Holland, Amsterdam, New York, Oxford, Tokyo, 1990.

3. M. Böhm, On a nonhomogeneous Bingham fluid, J. Differ. Equations, 60(2):259-284, 1985.

4. E. Fernández-Cara, F. Guillén, and R.R. Ortega, Some theoretical results for visco-plastic and dilatant fluids with variable density, Nonlinear Anal., Theory Methods Appl., 28(6):1079-1100, 1997.

5. Y. Giga and S. Takahashi, On global weak solutions of the nonstationary two-phase Stokes flow, SIAM J. Math. Anal., 25(3):876-893, 1994.

6. W.A. Kirk and B. Sims, Handbook of Metric Fixed Point Theory, Kluwer Academic, London, 2001.

7. O.A. Ladyzhenskaya, Mathematical Problems in Viscous Incompressible Fluid Dynamics, Nauka, Moscow, 1970.

8. O.A. Ladyzhenskaya and V.A. Solonnikov, Unique solvability of an initial- and boundary-value problem for viscous incompressible nonhomogeneous fluids, J. Sov. Math., 9(5):697-749, 1978.

9. O.A. Ladyzhenskaya, V.A. Solonnikov, and N.N. Ural'ceva, Linear and Quasi-Linear Parabolic Equations, Nauka, Moscow, 1967.

10. A. Meirmanov, The Muskat problem for a viscoelastic filtration, Interfaces Free Bound., 13(4):463-484, 2011.

11. A. Meirmanov, O. Galtsev, and R.N. Zimin, Displacement of oil by water in a single elastic capillary, Bound. Value Probl., 2017:83, 2017.

12. A. Meirmanov, R. Zimin, and K. Shiyapov, The Muskat problem at the microscopic level for a single capillary, Boundary Value probl., 2015:71, 2015.

13. A. Nouri and F. Poupaud, An existence theorem for the multifluid Navier-Stokes problem, J. Differ. Equations, 122(1):71-88, 1995.

14. E. Sanchez-Palencia, Non-Homogeneous Media and Vibration Theory, Lect. Notes Phys., Vol. 129, Springer, Berlin, 1980.

15. J. Simon, Nonhomogeneous viscous incompressible fluids: Existence of velocity, density, and pressure, SIAM J. Math. Anal., 21(5):1093-1117, 1990.

16. C.-S. Yih, Dynamics of Nonhomogeneous Fluids, Collier-Macmillan, London, 1965.

17. W. Zheng, On Friedrichs-Poincaré-type inequalities, J. Math. Anal. Appl., 304(2):542-551, 2005. 